\title{
Inner- and outer-sphere roles of ruthenium phosphido complexes in the hydrophosphination of alkenes
}

Roman G. Belli, Krista M. E. Burton, Stephanie A. Rufh, Robert McDonald $\ddagger$, and Lisa Rosenberg*

Department of Chemistry, University of Victoria, P. O. Box 3065, Victoria, BC, Canada V8W 3V6; ‡ X-ray Crystallography Laboratory, Department of Chemistry, University of Alberta, Edmonton, Alberta, Canada, T6G 2G2

\section{lisarose@uvic.ca}

\begin{tabular}{|c|c|}
\hline Contents & page \\
\hline${ }^{31} \mathrm{P}\left\{{ }^{1} \mathrm{H}\right\}$ NMR data for metallacycles $3 \mathbf{a}-\mathbf{c}$ & 2 \\
\hline $\begin{array}{l}\text { Metallacycle protonolysis experiments and }{ }^{31} \mathrm{P}\left\{{ }^{1} \mathrm{H}\right\} \text { NMR data for } \\
\text { complexes } \mathbf{4 a - c}\end{array}$ & 2 \\
\hline Characterization of $\mathbf{4 a}$ and NMR spectra of its reaction with $\mathrm{HPCy}_{2}$ & 4 \\
\hline Crystallographic data for complexes 6 and 7 & 7 \\
\hline Table of ${ }^{1} \mathrm{H}$ NMR data for $\mathbf{3 c}, \mathbf{6}, \mathbf{7}$ and $\mathbf{9}$ & 9 \\
\hline Table of ${ }^{13} \mathrm{C}\left\{{ }^{1} \mathrm{H}\right\}$ NMR data for $\mathbf{3 c}, \mathbf{6}, 7$ and 9 & 11 \\
\hline NMR spectra for complexes $\mathbf{3 c}, \mathbf{6}, \mathbf{7}$ and $\mathbf{9}$ & 13 \\
\hline $\begin{array}{l}\text { Representative } 145.78 \mathrm{MHz}{ }^{31} \mathrm{P}\left\{{ }^{1} \mathrm{H}\right\} \quad \mathrm{NMR} \text { spectra of catalyst } \\
\text { screening reactions, and associated control reactions, carried out in } 3: 1 \\
\mathrm{THF} / d_{6} \text {-benzene }\end{array}$ & 21 \\
\hline $\begin{array}{l}\text { Expansions of }{ }^{31} \mathrm{P}\left\{{ }^{1} \mathrm{H}\right\} \text { NMR spectra showing catalyst resting states } \\
\text { for the reactions of acrylonitrile with } \mathrm{HPPh}_{2}\end{array}$ & 28 \\
\hline${ }^{31} \mathrm{P}\left\{{ }^{1} \mathrm{H}\right\}$ NMR spectra for thermolysis of complex 9 & 32 \\
\hline${ }^{1} \mathrm{H} /{ }^{31} \mathrm{P} \mathrm{HMBC}$ NMR characterization of $\mathrm{Cy}_{2} \mathrm{PCH}_{2} \mathrm{CH}_{2} \mathrm{CN}$ & 33 \\
\hline
\end{tabular}




\section{${ }^{31} \mathbf{P}\left\{{ }^{1} \mathrm{H}\right\}$ NMR data for metallacycles 3a-c:}

Table S1. ${ }^{31} \mathrm{P}\left\{{ }^{1} \mathrm{H}\right\}$ NMR data for metallacycles 3a-c: $\delta(\mathrm{ppm})\left(\right.$ multiplicity, $\left.{ }^{2} \mathrm{~J}_{\mathrm{PP}}(\mathrm{Hz})\right)$.

\begin{tabular}{|c|c|c|c|}
\hline Complex & & $-\mathrm{PCy}_{2}$ & $\mathbf{P P h}_{3}$ \\
\hline \multirow[t]{2}{*}{$\mathrm{Ru}\left(\eta^{5}\right.$-indenyl $)\left(\kappa^{2}-\mathrm{Bu}^{n} \underline{\mathrm{C}} \mathrm{HCH}_{2} \mathrm{PCy}_{2}\right)\left(\mathrm{PPh}_{3}\right)^{a, b}$} & syn-3a & $-15.6(d, 22)$ & $63.8(d)$ \\
\hline & anti-3a & $-10.5(\mathrm{~d}, 28)$ & $60.3(d)$ \\
\hline \multirow[t]{2}{*}{$\mathrm{Ru}\left(\eta^{5}\right.$-indenyl $)\left(\kappa^{2}-(\mathrm{NC}) \underline{\mathrm{C}} \mathrm{HCH}_{2} \underline{\mathrm{PCy}}_{2}\right)\left(\mathrm{PPh}_{3}\right)^{a, b}$} & syn-3b & $-13.3(\mathrm{~d}, 24)$ & $61.2(d)$ \\
\hline & anti-3b & $-8.7(d, 26)$ & 56.0 \\
\hline \multirow[t]{2}{*}{$\mathrm{Ru}\left(\eta^{5}\right.$-indenyl $)\left(\kappa^{2}-\mathrm{PhCHCH}_{2} \underline{\mathrm{PC}} \mathrm{Cy}_{2}\right)\left(\mathrm{PPh}_{3}\right)^{c}$} & syn-3c & $-21.9(\mathrm{~d}, 21)$ & $63.6(d)$ \\
\hline & anti-3c & $-10.3(\mathrm{~d}, 27)$ & $59.0(d)$ \\
\hline
\end{tabular}

${ }^{a}$ Previously reported. ${ }^{1}{ }^{b} 202.5 \mathrm{MHz}, d_{6}$-benzene. ${ }^{c} 145.8 \mathrm{MHz}, d_{6}$-benzene.

\section{Metallacycle protonolysis experiments and ${ }^{31} \mathrm{P}\{1 \mathrm{H}\}$ NMR data for complexes 4a-c:}

Pyridine hydrochloride One equiv of a 5.84 M solution of pyridine hydrochloride in acetonitrile was added via a microsyringe to a solution of the metallacycle $(15-20 \mathrm{mg})$ in $d_{6}$-benzene in a J Young NMR tube.

3a: The metallacycle was completely consumed within 15 min, giving $\mathbf{4 a}$, as well as some $\mathbf{1}$, and a second product tentatively assigned as the bis(phosphine) complex $\mathrm{Ru}\left(\mathrm{n}^{5}\right.$ indenyl) $\mathrm{Cl}\left\{\mathrm{PCy}_{2}\left(\mathrm{CH}_{2} \mathrm{CH}_{2} \mathrm{Bu}^{n}\right)\right\}_{2}(\mathrm{~d} @ 47.1 \mathrm{ppm})$.

3b: Peaks due to $\mathbf{4 b}$ were detected immediately and grew in slowly over $24 \mathrm{~h}$.

3c: Peaks due to $\mathbf{4 c}$ began to appear within hours of mixing.

2,6-Lutidine hydrochloride A solution of one equiv 2,6-lutidine hydrochloride in $d_{1}$-chloroform was added to the solid metallacycle (15-20 mg) in a J Young NMR tube.

3a: The starting material was completely consumed within 15 minutes, giving principally $4 \mathbf{a}$ and trace amounts of apparent ligand redistribution products. These increased in concentration over $24 \mathrm{~h}$.

3b: Peaks due to $\mathbf{4 b}$ were detected immediately and grew in quickly.

3c: Peaks due to $\mathbf{4 c}$ appeared gradually, along with other sets of doublets that have not yet been assigned.

Triethylamine hydrochloride A suspension of 20 equiv triethylamine hydrochloride in $d_{6}$-benzene was added to the solid metallacycle $(15-20 \mathrm{mg})$ in a J Young NMR tube. The tube was placed in a sonicator to completely dissolve the triethylamine hydrochloride.

3a: Peaks due to 4a grew in slowly over 24 h. Before reaction was complete, signals due to 1 (d @) $47.8 \mathrm{ppm})$ and (tentatively) the bis(phosphine) complex Ru( $\mathrm{n}^{5}$-indenyl $) \mathrm{Cl}\left\{\mathrm{PCy}_{2}\left(\mathrm{CH}_{2} \mathrm{CH}_{2} \mathrm{R}\right)\right\}_{2}(\mathrm{~d} @$ $47.1 \mathrm{ppm}$ ) had also appeared.

${ }^{1}$ Derrah, E. J., Pantazis, D. A., McDonald, R., Rosenberg, L. Angew. Chem. Int. Ed. 2010, 49, 3367. 
3b: No reaction was observed after $24 \mathrm{~h}$.

3c: No reaction was observed after $24 \mathrm{~h}$.

Table S2. $121.46 \mathrm{MHz}{ }^{31} \mathrm{P}\left\{{ }^{1} \mathrm{H}\right\} \mathrm{NMR}$ data for $\mathrm{Ru}\left(\mathrm{n}^{5}\right.$-indenyl $) \mathrm{Cl}\left\{\mathrm{PCy}_{2}\left(\mathrm{CH}_{2} \mathrm{CH}_{2} \mathrm{R}^{\prime}\right)\right\} \mathrm{PPh}_{3}, \mathbf{4 a - c}: \delta$ (ppm) (multiplicity, ${ }^{2} \mathrm{~J}_{\mathrm{PP}}(\mathrm{Hz})$ ).

\begin{tabular}{|c|c|c|c|c|}
\hline Complex (R') & $\mathbf{P C y}_{2}\left(\mathbf{C H}_{2} \mathbf{C H}_{2} \mathbf{R}^{\prime}\right)$ & $\mathbf{P P h}_{\mathbf{3}}$ & $\mathbf{P C y}_{\mathbf{2}}\left(\mathbf{C H}_{2} \mathbf{C H}_{2} \mathbf{R}^{\prime}\right)$ & $\mathbf{P P h}_{\mathbf{3}}$ \\
\hline & \multicolumn{2}{|c|}{$d_{6}$-benzene } & \multicolumn{2}{|c|}{$d_{1}$-chloroform } \\
\hline $\mathbf{4 a}\left(\mathrm{Bu}^{n}\right)$ & $41.0(\mathrm{~d}, 35)$ & $48.3(\mathrm{~d})$ & $43.0(\mathrm{~d}, 34)$ & $46.2(\mathrm{~d})$ \\
\hline $\mathbf{4 b}(\mathrm{CN})$ & $41.0(\mathrm{~d}, 35)$ & $51.2(\mathrm{~d})$ & $38.6(\mathrm{~d}, 36)$ & $50.5(\mathrm{~d})$ \\
\hline $\mathbf{4 c}(\mathrm{Ph})$ & $41.4(\mathrm{~d}, 35)$ & $47.6(\mathrm{~d})$ & $43.2(\mathrm{~d}, 34)$ & $45.5(\mathrm{~d})$ \\
\hline
\end{tabular}




\section{Characterization of $4 \mathrm{a}$ and NMR spectra of its reaction with $\mathrm{HPCy}_{2}$ :}

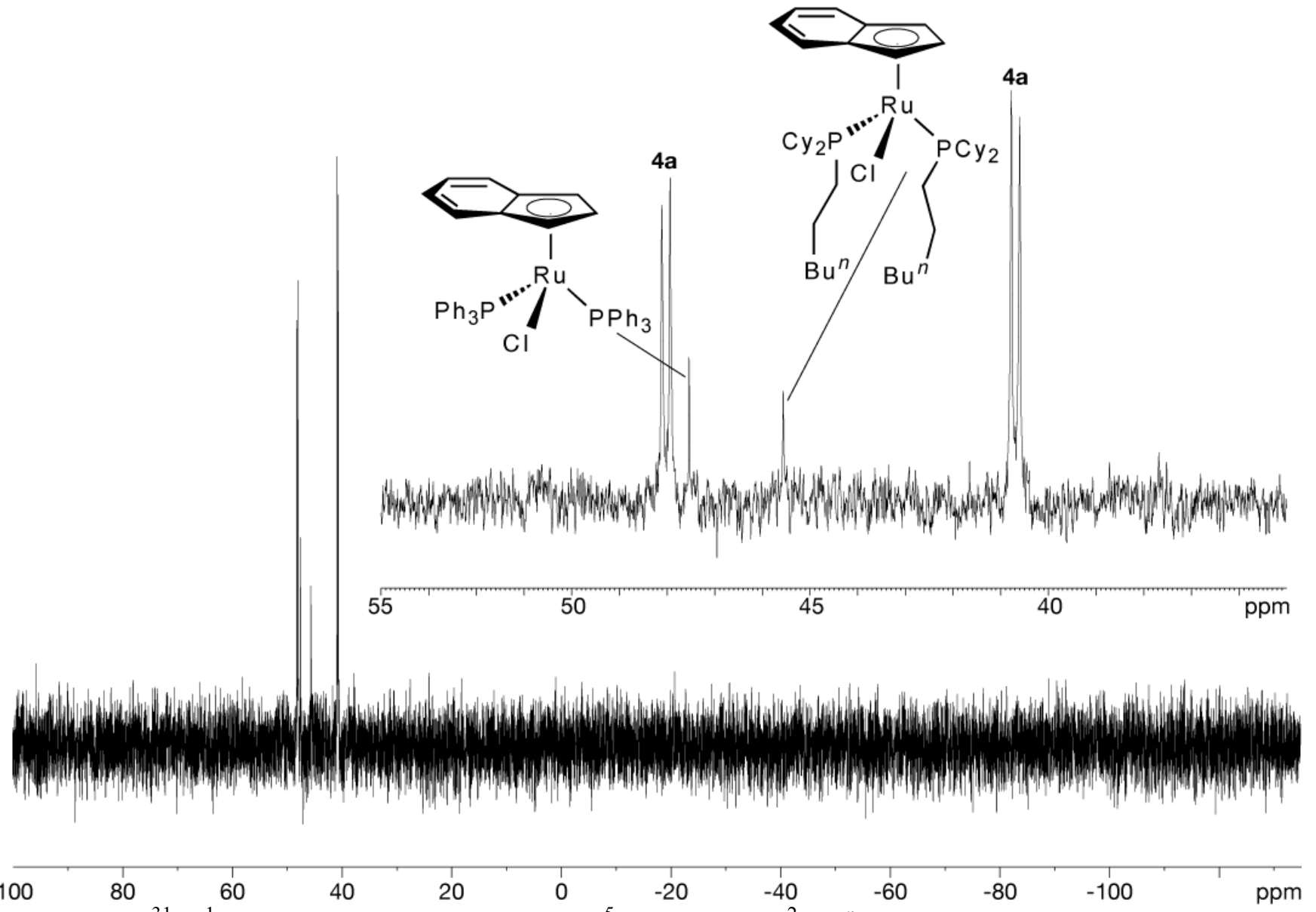

Figure S1. ${ }^{31} \mathrm{P}\left\{{ }^{1} \mathrm{H}\right\}$ NMR spectrum for $\mathrm{Ru}\left(\eta^{5}\right.$-indenyl $) \mathrm{Cl}\left(\kappa^{2}-\mathrm{Bu}^{n} \mathrm{CH}_{2} \mathrm{CH}_{2} \mathrm{PCy}_{2}\right)\left(\mathrm{PPh}_{3}\right)$, 4a, isolated from addition of pyridine hydrochloride to metallacycle 3a, in $d_{6}$-benzene. Products due to ligand redistribution are indicated. 

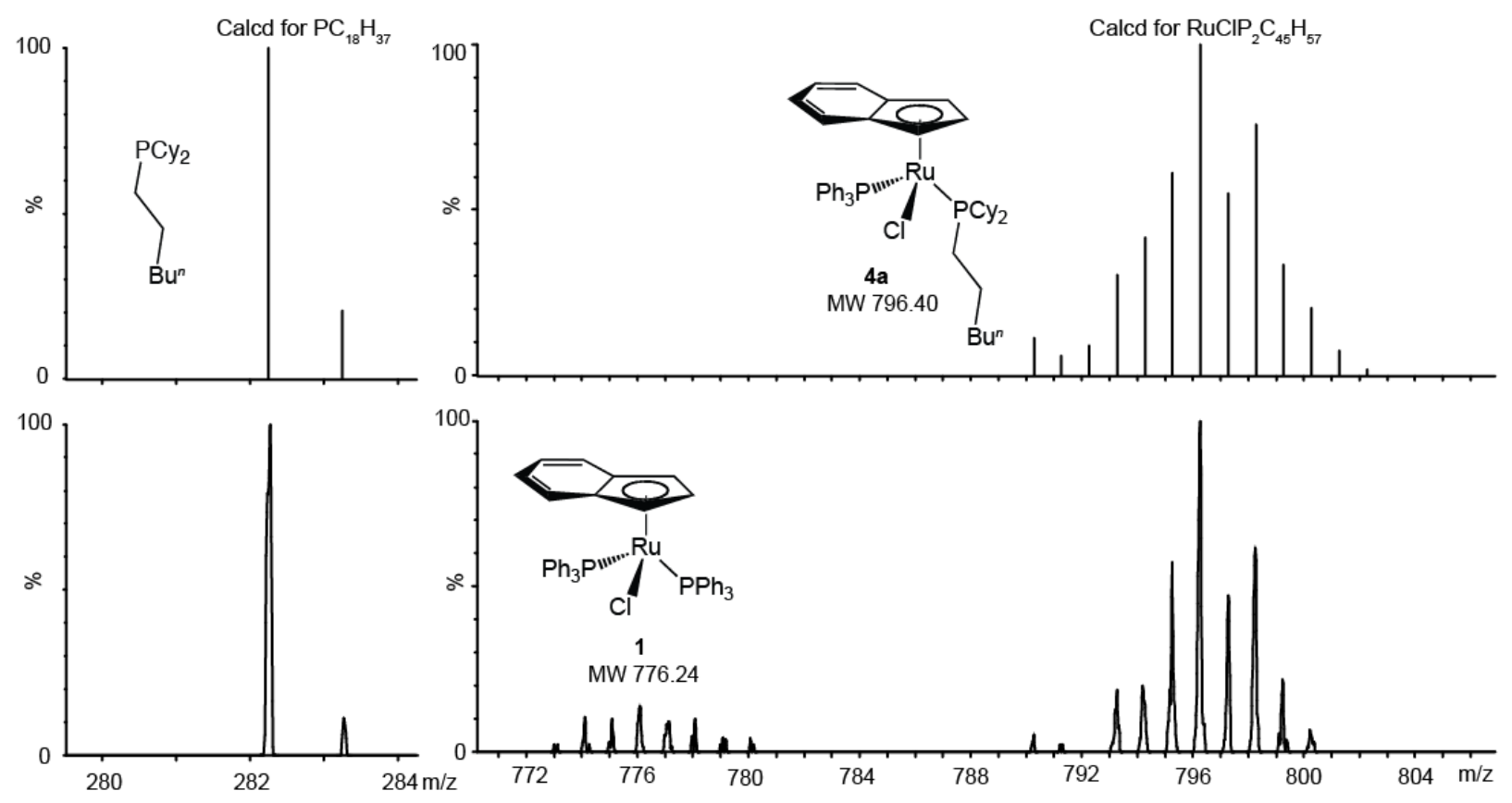

Figure S2. Top: calculated isotope patterns for $\mathbf{4 a}$ and for the free phosphine $\mathrm{PCy}_{2}($ hexyl). Bottom: experimental Liquid Introduction Field Desorption Ionization (LIFDI) MS data for a sample of 4a in acetone/toluene. 


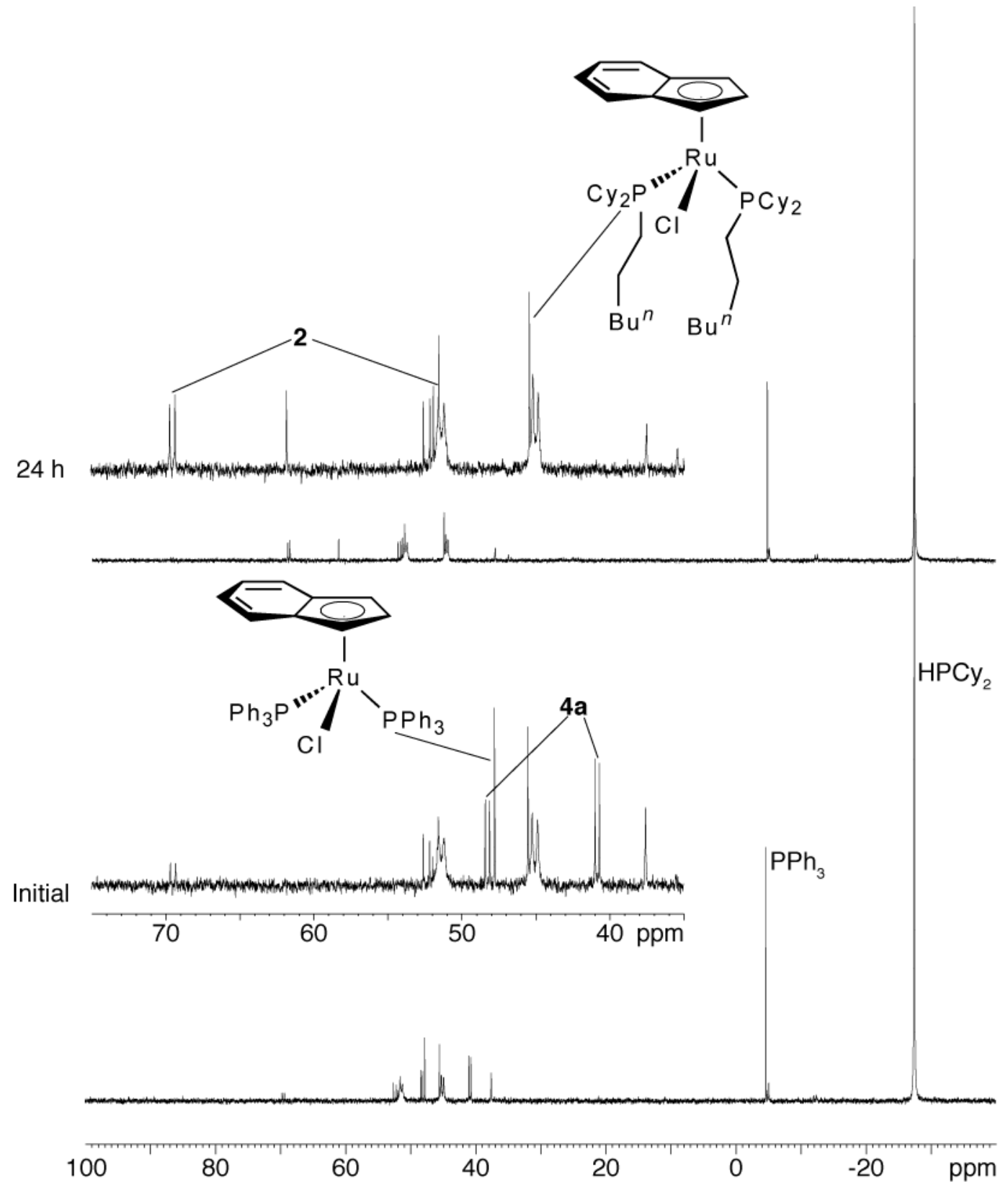

Figure S3. $121.44 \mathrm{MHz}{ }^{31} \mathrm{P}\left\{{ }^{1} \mathrm{H}\right\}$ NMR spectra showing the reaction of excess $\mathrm{HPCy}_{2}$ with $\mathrm{Ru}\left(\eta^{5}\right.$ indenyl $) \mathrm{Cl}\left(\kappa^{2}-\mathrm{Bu}^{n} \mathrm{CH}_{2} \mathrm{CH}_{2} \mathrm{PCy}_{2}\right)\left(\mathrm{PPh}_{3}\right)$, 4a, generated in situ in $d_{6}$-benzene. Several unidentified, mixed phosphine products are observed, as well as complex 2, and products due to ligand redistribution. 


\section{Crystallographic data for complexes 6 and 7:}

Table S3. Crystallographic experimental details

\begin{tabular}{|c|c|c|}
\hline compound & 6 & 7 \\
\hline formula & $\mathrm{C}_{91} \mathrm{H}_{57} \mathrm{BF}_{20} \mathrm{NP}_{2} \mathrm{Ru}$ & $\mathrm{C}_{70} \mathrm{H}_{38} \mathrm{BF}_{20} \mathrm{NP}_{2} \mathrm{Ru}$ \\
\hline formula weight & 1718.19 & 1446.83 \\
\hline crystal dimensions $(\mathrm{mm})$ & $0.24^{\prime} 0.22^{\prime} 0.15$ & $0.37^{\prime} 0.22^{\prime} 0.21$ \\
\hline crystal system & triclinic & \begin{tabular}{|l|} 
triclinic \\
\end{tabular} \\
\hline space group & $P \overline{1}$ (No. 2) & $P \overline{1}($ No. 2$)$ \\
\hline$a(\AA)$ & $16.0400(6)^{a}$ & $13.0253(5)^{b}$ \\
\hline$b(\AA)$ & $16.8124(6)$ & $13.4007(5)$ \\
\hline$c(\AA)$ & $16.8412(6)$ & $18.4817(7)$ \\
\hline$\alpha(\operatorname{deg})$ & $104.6950(4)$ & $100.0631(5)$ \\
\hline$\beta(\operatorname{deg})$ & $107.8349(4)$ & $105.0829(5)$ \\
\hline$\gamma(\operatorname{deg})$ & $108.4208(4)$ & $94.3895(5)$ \\
\hline$V\left(\AA^{3}\right)$ & $3780.4(2)$ & $3041.5(2)$ \\
\hline$Z$ & 2 & 2 \\
\hline$\rho_{\text {calcd }}\left(\mathrm{g} \mathrm{cm}^{-3}\right)$ & 1.509 & 1.580 \\
\hline$\mu\left(\mathrm{mm}^{-1}\right)$ & 0.349 & 0.417 \\
\hline diffractometer & \multicolumn{2}{|c|}{ Bruker PLATFORM/APEX II CCD ${ }^{c}$} \\
\hline radiation $(\lambda[\AA])$ & \multicolumn{2}{|c|}{ graphite-monochromated Mo Ka $(0.71073)$} \\
\hline temperature $\left({ }^{\circ} \mathrm{C}\right)$ & \multicolumn{2}{|c|}{-80} \\
\hline scan type & \multicolumn{2}{|c|}{$\omega$ scans $\left(0.3^{\circ}\right)(15 \mathrm{~s}$ exposures $)$} \\
\hline $2 \theta_{\max }(\operatorname{deg})$ & 56.87 & 56.76 \\
\hline total data collected & $\begin{array}{l}35924(-21 \leq h \leq 21,-22 \leq k \leq 22,- \\
22 \leq l \leq 22)\end{array}$ & $\begin{array}{l}27382(-17 \leq h \leq 17,-17 \leq k \leq \\
17,-24 \leq l \leq 24)\end{array}$ \\
\hline independent reflections & $18521\left(R_{\text {int }}=0.0249\right)$ & $14662\left(R_{\text {int }}=0.0198\right)$ \\
\hline $\begin{array}{l}\text { observed reflections } \\
(N O)\end{array}$ & $15091\left[F_{\mathrm{o}}^{2} \geq 2 \sigma\left(F_{\mathrm{o}}^{2}\right)\right]$ & $12370\left[F_{\mathrm{o}}^{2} \geq 2 \sigma\left(F_{\mathrm{o}}^{2}\right)\right]$ \\
\hline structure solution method & intrinsic phasing $($ SHELXT-2014d $)$ & $\begin{array}{l}\text { Patterson/structure expansion } \\
\left(D I R D I F-2008^{e}\right)\end{array}$ \\
\hline refinement method & \multicolumn{2}{|c|}{ full-matrix least-squares on $F^{2}(S H E L X L-2014 f)$} \\
\hline $\begin{array}{l}\text { absorption correction } \\
\text { method }\end{array}$ & \multicolumn{2}{|c|}{ Gaussian integration (face-indexed) } \\
\hline $\begin{array}{l}\text { range of transmission } \\
\text { factors }\end{array}$ & $1.0000-0.9187$ & $0.9823-0.9063$ \\
\hline data/restraints/parameters & $18521 / 0 / 1000$ & $14662 / 0 / 860$ \\
\hline $\begin{array}{l}\text { goodness-of-fit }(S) \text { [all } \\
\text { data] }\end{array}$ & 1.047 & 1.050 \\
\hline$R_{1}\left[F_{\mathrm{O}}^{2} \geq 2 \sigma\left(F_{\mathrm{O}}^{2}\right)\right]$ & 0.0377 & 0.0360 \\
\hline$w R_{2}$ [all data] & 0.0970 & 0.0920 \\
\hline $\begin{array}{l}\text { largest difference peak } \\
\text { and hole }\end{array}$ & 0.793 and -0.559 e $\AA^{-3}$ & 0.895 and -0.742 e $\AA^{-3}$ \\
\hline
\end{tabular}


${ }^{a}$ Obtained from least-squares refinement of 9994 reflections with $4.60^{\circ}<2 \theta<52.24^{\circ}$. ${ }^{b}$ Obtained from least-squares refinement of 9720 reflections with $4.52^{\circ}<2 \theta<51.98^{\circ}$. ${ }^{c}$ Programs for diffractometer operation, data collection, data reduction and absorption correction were those supplied by Bruker. ${ }^{d}$ Sheldrick, G. M. Acta Crystallogr. 2015, A71, 3-8. ${ }^{e}$ Beurskens, P. T.; Beurskens, G.; de Gelder, R.; Smits, J. M. M.; Garcia-Granda, S.; Gould, R. O. (2008). The DIRDIF-2008 program system. Crystallography Laboratory, Radboud University Nijmegen, The Netherlands. fSheldrick, G. M. Acta Crystallogr. 2015, C71, 3-8. 
Table S4. $500.13 \mathrm{MHz}{ }^{1} \mathrm{H}$ NMR data for complexes 3c, 6, 7, and 9 at 300K: $\delta$ in ppm (multiplicity, RI, $\mathrm{J}_{\text {avg }}$ or $\omega_{1 / 2}$ in $\mathrm{Hz}$, assignment). (See Fig. S7 below for structure illustrating locations of metallacycle protons $\mathrm{H}_{\mathrm{a}-\mathrm{d}}$.)

\begin{tabular}{|c|c|c|c|c|c|c|}
\hline Compound & \multicolumn{4}{|l|}{$\mathrm{C}_{9} \mathrm{H}_{7}$} & $\mathbf{P P h}_{3}$ & Other \\
\hline $\begin{array}{l}\text { syn-3c } \\
d_{8} \text {-toluene }\end{array}$ & $\begin{array}{l}7.40\left(\mathrm{~d},{ }^{3} \mathrm{~J}_{\mathrm{HH}} 8,\right. \\
1 \mathrm{H}) 5.80 \quad(\mathrm{~d}, \\
\left.{ }^{3} \mathrm{~J}_{\mathrm{HH}} 8,1 \mathrm{H}\right)\end{array}$ & $\begin{array}{lr}6.89-6.80 \quad \text { (om, } \\
5 \mathrm{H} \text {, overlaps } \\
\mathrm{H}_{0}, \quad \mathrm{H}_{\mathrm{m}} \text { of } \\
\text { styrenyl } \mathrm{Ph})\end{array}$ & $\begin{array}{l}4.22-4.19 \\
(\mathrm{~m}, 1 \mathrm{H})\end{array}$ & $\begin{array}{l}4.94 \quad(\mathrm{~s}, \\
1 \mathrm{H}) \quad 4.04 \\
(\mathrm{~s}, 1 \mathrm{H})\end{array}$ & $\begin{array}{l}\text { Ho } 7.84-7.54 \\
(\mathrm{br} \mathrm{m}, 5 \mathrm{H}) \\
\mathrm{H}_{\mathrm{m}}, \mathrm{H}_{\mathrm{p}} 6.99- \\
6.88 \quad \text { (om, } \\
9 \mathrm{H} \text {, overlaps } \\
\text { with } \mathrm{H}_{6} \text { anti) }\end{array}$ & 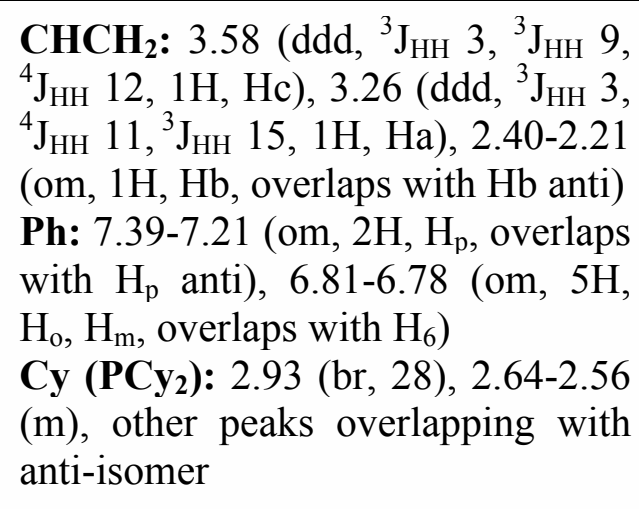 \\
\hline $\begin{array}{l}\text { anti-3c } \\
d_{8} \text {-toluene }\end{array}$ & $\begin{array}{l}7.60\left(\mathrm{~d},{ }^{3} \mathrm{~J}_{\mathrm{HH}} 8,\right. \\
1 \mathrm{H}) 5.99(\mathrm{~d}, \\
\left.{ }^{3} \mathrm{~J}_{\mathrm{HH}} 8,1 \mathrm{H}\right)\end{array}$ & $\begin{array}{l}6.91 \quad\left(\mathrm{t}, \quad{ }^{3} \mathrm{~J}_{\mathrm{HH}} \quad 8,\right. \\
1 \mathrm{H}) \\
6.59 \quad\left(\mathrm{t}, \quad{ }^{3} \mathrm{~J}_{\mathrm{HH}} \quad 7,\right. \\
1 \mathrm{H})\end{array}$ & $\begin{array}{l}5.73-5.70 \\
(\mathrm{~m}, 1 \mathrm{H})\end{array}$ & $\begin{array}{l}5.58 \quad(\mathrm{~s}, \\
1 \mathrm{H}) \quad 3.94 \\
(\mathrm{~s}, 1 \mathrm{H})\end{array}$ & $\begin{array}{l}\mathrm{H}_{\mathrm{o}}, \quad \mathrm{H}_{\mathrm{m}}, \quad \mathrm{H}_{\mathrm{p}} \\
7.17-7.00 \\
(\mathrm{om}, 15 \mathrm{H})\end{array}$ & 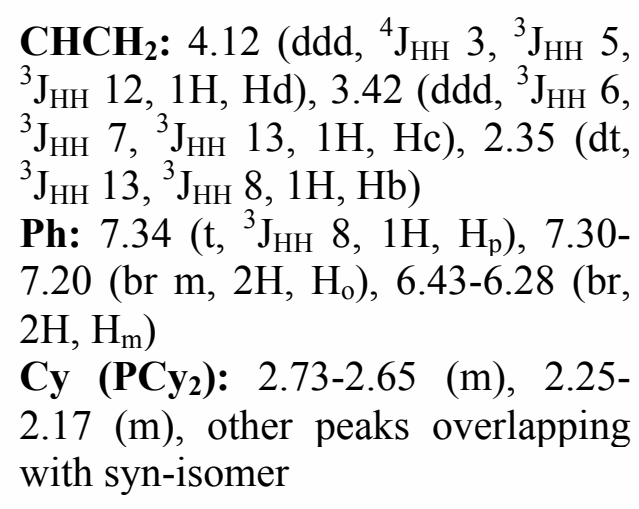 \\
\hline $\begin{array}{l}6 \\
d_{2^{-}} \\
\text {dichloromethane }\end{array}$ & $\begin{array}{l}7.14 \quad\left(\mathrm{~d}, \quad{ }^{3} \mathrm{~J}_{\mathrm{HH}}\right. \\
7.3,2 \mathrm{H})\end{array}$ & $\begin{array}{l}7.38\left(\mathrm{t},{ }^{3} \mathrm{~J}_{\mathrm{HH}} 7.3,\right. \\
2 \mathrm{H})\end{array}$ & $4.90(\mathrm{~s}, 1 \mathrm{H})$ & $\begin{array}{l}4.52 \\
2 \mathrm{H})\end{array}$ & $\begin{array}{l}\mathrm{H}_{\mathrm{o}} 7.24 \quad(\mathrm{t}, \\
{ }^{3} \mathrm{~J}_{\mathrm{HH}} \quad 7.3, \\
12 \mathrm{H}) \\
\mathrm{H}_{\mathrm{m}} 6.98(\mathrm{br},\end{array}$ & $\begin{array}{l}\text { NCPh: } \\
\mathrm{H}_{\mathrm{o}} 7.31(\mathrm{~m}, 2 \mathrm{H}) \\
\mathrm{H}_{\mathrm{m}} 6.83(\mathrm{~m}, 2 \mathrm{H}) \\
\mathrm{H}_{\mathrm{p}} 7.64\left(\mathrm{t},{ }^{3} \mathrm{~J}_{\mathrm{HH}} 7.8,1 \mathrm{H}\right)\end{array}$ \\
\hline
\end{tabular}




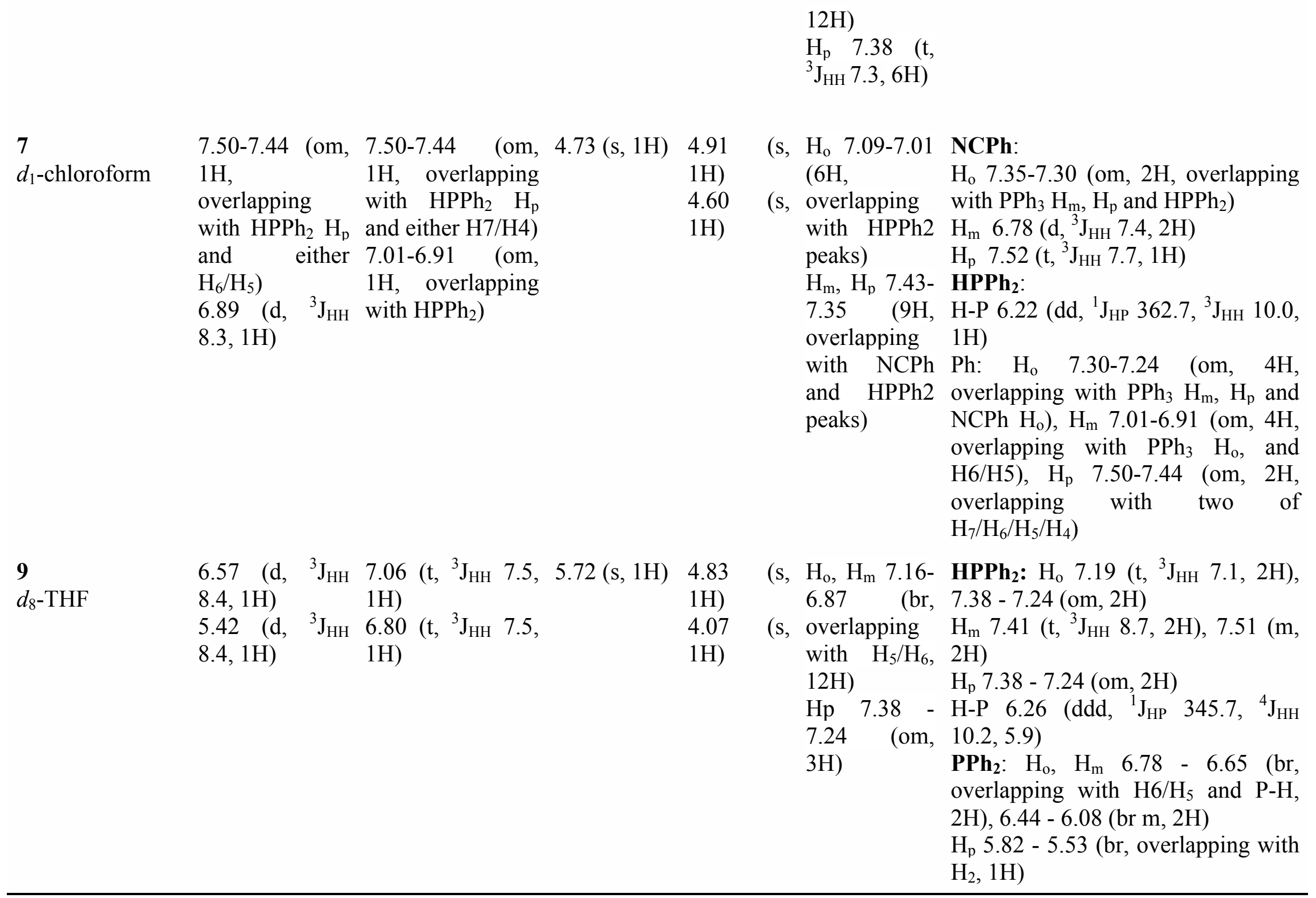


Table S5. $125.77 \mathrm{MHz}{ }^{13} \mathrm{C}\left\{{ }^{1} \mathrm{H}\right\}$ NMR data for complexes 3c, 6, 7 and 9 at $300 \mathrm{~K}: \delta$ in ppm (multiplicity, RI, $\mathrm{J}_{\mathrm{avg}}$ or $\omega_{1 / 2}$ in $\mathrm{Hz}$, assignment). See Eqn 1 in article for structure illustrating locations of $\mathrm{C} \alpha$ and $\mathrm{C} \beta$ in metallacycles.

\begin{tabular}{|c|c|c|c|c|c|c|c|c|}
\hline \multirow[t]{2}{*}{ Compound } & \multicolumn{6}{|l|}{$\mathrm{C}_{9} \mathrm{H}_{7}$} & \multirow[t]{2}{*}{$\mathbf{P P h}_{3}$} & \multirow[t]{2}{*}{ Other } \\
\hline & $C_{6}, C_{5}$ & $\mathbf{C}_{7}, \mathbf{C}_{4}$ & $\mathbf{C}_{7 \mathbf{a}}, \mathbf{C}_{4 a}$ & $\begin{array}{l}\Delta \delta\left(C_{7 \mathbf{a}}\right. \\
\left.\mathbf{C}_{\mathbf{3 a}}\right)^{a}\end{array}$ & $\mathbf{C}_{2}$ & $\mathbf{C}_{3}, \mathbf{C}_{1}$ & & \\
\hline $\begin{array}{l}\text { syn-3c } \\
d_{8} \text {-toluene }\end{array}$ & $\begin{array}{l}121.6(\mathrm{~s}) \\
124.0(\mathrm{~s})\end{array}$ & $\begin{array}{l}126.1 \\
(\mathrm{~s}) \\
121.4 \\
(\mathrm{~s})\end{array}$ & $\begin{array}{l}109.2(\mathrm{~s}) \\
104.6(\mathrm{~s})\end{array}$ & -23.8 & $\begin{array}{l}103.0 \\
(\mathrm{~s})\end{array}$ & $\begin{array}{l}68.5 \\
\left.{ }^{2} \mathrm{~J}_{\mathrm{CP}} 9\right) \\
70.6 \\
\left.{ }^{2} \mathrm{~J}_{\mathrm{CP}} 9\right)\end{array}$ & $\begin{array}{ll}\mathrm{C}_{\mathrm{i}} & 135.5 \\
(\mathrm{br}, 29) \\
\mathrm{C}_{\mathrm{o}} & 134.2 \\
\left(\mathrm{~d},{ }^{2} \mathrm{~J}_{\mathrm{CP}}\right. & 11) \\
\mathrm{Cm} & 125.4 \\
(\mathrm{~s}) & \\
\mathrm{C}_{\mathrm{p}} & 129.0 \\
(\mathrm{~s}) & \end{array}$ & $\begin{array}{l}\mathbf{C H C H}_{2}: 39.8\left(\mathrm{~d},{ }^{1} \mathrm{~J}_{\mathrm{CP}} 29, \mathrm{C} \beta\right), \\
0.7\left(\mathrm{dd},{ }^{2} \mathrm{~J}_{\mathrm{CP}} 828, \mathrm{C} \alpha\right) \\
\text { Ph: } 162.0\left(\mathrm{~s}, \mathrm{C}_{\mathrm{i}}\right), 127.9-127.1 \\
\left.\text { (br om, } \mathrm{C}_{\mathrm{o}}\right), 124.6\left(\mathrm{~s}, \mathrm{C}_{\mathrm{p}}\right), 121.9 \\
\left(\mathrm{~s}, \mathrm{C}_{\mathrm{m}}\right) \\
\text { PC } \mathbf{y}_{2}: \\
\text { PCH } 48.3\left(\mathrm{dd},{ }^{1} \mathrm{~J}_{\mathrm{CP}} 3{ }^{2} \mathrm{~J}_{\mathrm{CP}} 12\right), \\
43.3\left(\mathrm{~d},{ }^{1} \mathrm{~J}_{\mathrm{CP}} 4\right) \\
\text { Others } 31.2(\mathrm{~s}), 30.3\left(\mathrm{~d},{ }^{2} \mathrm{~J}_{\mathrm{CP}}\right. \\
8), 30.2(\mathrm{~s}), 30.1(\mathrm{~s}), 28.9(\mathrm{~d}, \\
\left.{ }^{2} \mathrm{~J}_{\mathrm{CP}} 7\right), 28.7\left(\mathrm{~d},{ }^{2} \mathrm{~J}_{\mathrm{CP}} 11\right), 27.7 \\
\left(\mathrm{~d},{ }^{2} \mathrm{~J}_{\mathrm{CP}} 7\right), 26.8(\mathrm{~s})\end{array}$ \\
\hline $\begin{array}{l}\text { anti-3c } \\
d_{8} \text {-toluene }\end{array}$ & $\begin{array}{l}121.5(\mathrm{~s}) \\
123.9(\mathrm{~s})\end{array}$ & $\begin{array}{l}124.3 \\
(\mathrm{~s}) \\
120.4 \\
(\mathrm{~s})\end{array}$ & $\begin{array}{l}110.7(\mathrm{~s}) \\
104.3(\mathrm{~s})\end{array}$ & -23.2 & $96.8(\mathrm{~s})$ & $\begin{array}{l}64.6 \\
\left.{ }^{2} \mathrm{~J}_{\mathrm{CP}} 11\right) \\
74.2 \\
\left.{ }^{2} \mathrm{~J}_{\mathrm{CP}} 11\right)\end{array}(\mathrm{d}$, & $\begin{array}{ll}\mathrm{C}_{\mathrm{i}} & 138.8 \\
(\mathrm{br}, 24) & \\
\mathrm{C}_{\mathrm{o}} & 133.6 \\
(\mathrm{~d}, 10) & \\
\mathrm{C}_{\mathrm{m}} & 128.3 \\
(\mathrm{~s}) & \\
\mathrm{C}_{\mathrm{p}} & 129.2 \\
(\mathrm{~s}) & \end{array}$ & 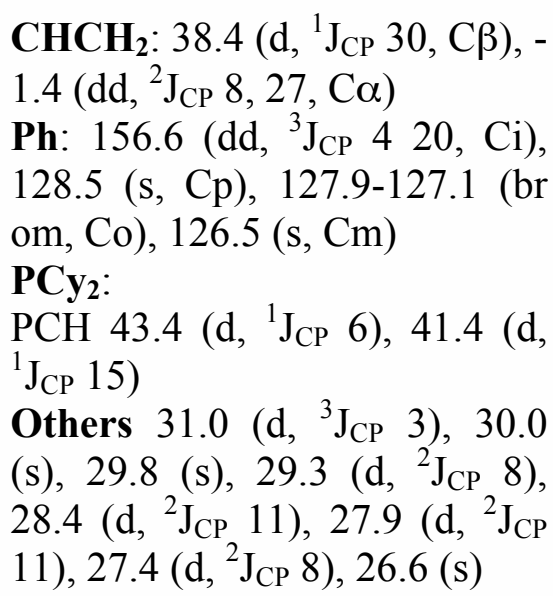 \\
\hline
\end{tabular}




\begin{tabular}{|c|c|c|c|c|c|c|c|c|}
\hline $\begin{array}{l}\mathbf{6} \\
d_{2-} \\
\text { dichloromethane }\end{array}$ & $131.8(\mathrm{~s})$ & $\begin{array}{l}129.7 \\
(\mathrm{~s})\end{array}$ & $109.6(\mathrm{~s})$ & -21.1 & $93.7(\mathrm{~s})$ & $69.0(\mathrm{~s})$ & $\begin{array}{l}\mathrm{C}_{\mathrm{i}} 134.5(\mathrm{~s}) \\
\mathrm{C}_{\mathrm{o}} 128.2(\mathrm{t}, \\
\left.{ }^{2} \mathrm{~J}_{\mathrm{CP}} 5\right) \\
\mathrm{C}_{\mathrm{m}} 133.3 \\
\left(\mathrm{t},{ }^{3} \mathrm{~J}_{\mathrm{CP}} 5\right) \\
\mathrm{C}_{\mathrm{p}} \quad 130.3 \\
(\mathrm{~s})\end{array}$ & 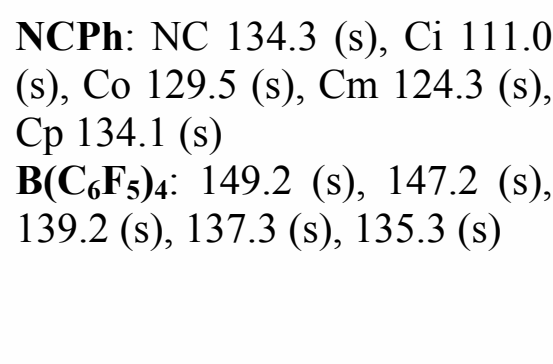 \\
\hline $\begin{array}{l}7 \\
d_{1} \text {-chloroform }\end{array}$ & $\begin{array}{l}131.7(\mathrm{~s}) \\
129.3(\mathrm{~s})\end{array}$ & $\begin{array}{l}129.0 \\
(\mathrm{~s}) \\
124.7 \\
(\mathrm{~s})\end{array}$ & $\begin{array}{l}109.5(\mathrm{~d}, \\
\left.{ }^{2} \mathrm{~J}_{\mathrm{CP}} 2\right) \\
107.7(\mathrm{~s})\end{array}$ & -22.1 & $93.0(\mathrm{~s})$ & $\begin{array}{l}67.2(\mathrm{~s}) \\
68.9 \\
\left.{ }^{2} \mathrm{~J}_{\mathrm{CP}} 6\right)\end{array}$ & $\begin{array}{l}\mathrm{C}_{\mathrm{i}} 132.5 \\
\mathrm{C}_{\mathrm{o}} \quad 133.1 \\
\left(\mathrm{~d},{ }^{2} \mathrm{~J}_{\mathrm{CP}} 11\right) \\
\mathrm{C}_{\mathrm{m}} \quad 128.8 \\
\left(\mathrm{~d},{ }^{3} \mathrm{~J}_{\mathrm{CP}} 10\right) \\
\mathrm{C}_{\mathrm{p}} \quad 131.0 \\
\left(\mathrm{~d},{ }^{4} \mathrm{~J}_{\mathrm{CP}} 2\right)\end{array}$ & $\begin{array}{l}\text { PPh }_{2} \mathbf{H}: \mathrm{C}_{\mathrm{i}} 132.4(\mathrm{~s}), 132.0(\mathrm{~s}), \\
\mathrm{C}_{\mathrm{o}} 132.9\left(\mathrm{~d},{ }^{2} \mathrm{~J}_{\mathrm{CP}} 10\right), 131.4(\mathrm{~d}, \\
\left.{ }^{2} \mathrm{~J}_{\mathrm{CP}} 10\right), \mathrm{C}_{\mathrm{m}} 129.1\left(\mathrm{~d},{ }^{3} \mathrm{~J}_{\mathrm{CP}} 10\right), \\
\mathrm{C}_{\mathrm{p}} 130.8\left(\mathrm{~d},{ }^{4} \mathrm{~J}_{\mathrm{CP}} 2\right) \\
\text { NCPh: NC } 127.7(\mathrm{~s}), \mathrm{C}_{\mathrm{i}} 110.5 \\
(\mathrm{~s}), \mathrm{C}_{\mathrm{o}} 128.0(\mathrm{~s}), \mathrm{C}_{\mathrm{m}} 123.6(\mathrm{~s}), \\
\mathrm{C}_{\mathrm{p}} 134.0(\mathrm{~s}) \\
\mathbf{B}\left(\mathbf{C}_{6} \mathbf{F}_{5}\right)_{4}: 149.2(\mathrm{~s}), 147.2(\mathrm{~s}), \\
139.2(\mathrm{~s}), 137.3(\mathrm{~s}), 135.3(\mathrm{~s})\end{array}$ \\
\hline $\begin{array}{l}9 \\
d_{8}-\mathrm{THF}\end{array}$ & $\begin{array}{l}123.3(\mathrm{~s}) \\
121.0(\mathrm{~s})\end{array}$ & $\begin{array}{l}126.0 \\
(\mathrm{~s}) \\
122.6 \\
(\mathrm{~s})\end{array}$ & $\begin{array}{l}114.5(\mathrm{~s}) \\
107.2(\mathrm{~s})\end{array}$ & -19.9 & $98.8(\mathrm{~s})$ & $\begin{array}{l}74.4 \\
\left.{ }^{2} \mathrm{~J}_{\mathrm{CP}} 11\right) \\
72.9 \\
\left.{ }^{2} \mathrm{~J}_{\mathrm{CP}} 9\right)\end{array}$ & $\begin{array}{l}\mathrm{C}_{\mathrm{i}} 133.5(\mathrm{~s}) \\
\mathrm{C}_{\mathrm{o}}, \quad \mathrm{C}_{\mathrm{m}} \\
127.0 \text { (br s, } \\
29) \\
\mathrm{C}_{\mathrm{p}} \quad 128.8 \\
(\text { br s, } 23)\end{array}$ & $\begin{array}{l}\text { HPPh }_{2} \mathrm{C}_{\mathrm{i}} 135.5(\mathrm{~s}), 135.2(\mathrm{~s}) \\
\mathrm{C}_{\mathrm{o}} 127.8(\mathrm{~s}), 127.9(\mathrm{~s}) \\
\mathrm{C}_{\mathrm{m}} 133.0(\mathrm{~s}), 130.0(\mathrm{~s}) \\
\mathrm{C}_{\mathrm{p}} 131.3(\mathrm{~s}), 128.5(\mathrm{~s}) \\
\text { PPh }_{2}: \mathrm{Ci} 139.9(\mathrm{~s}), 139.6(\mathrm{~s}) \\
\mathrm{C}_{\mathrm{o}} 127.9(\mathrm{~s}), 128.0(\mathrm{~s}) \\
\mathrm{C}_{\mathrm{m}} 133.1,133.0(\mathrm{~s}) \\
\mathrm{C}_{\mathrm{p}} 131.4(\mathrm{~s}), 128.7(\mathrm{~s})\end{array}$ \\
\hline
\end{tabular}

${ }^{a} \Delta \delta\left(C_{3 a, 7 a}\right)=\delta\left(C_{3 a, 7 a}(\eta\right.$-indenyl complex $\left.)\right)-\delta\left(C_{3 a, 7 a}(\eta\right.$-sodium indenyl $\left.)\right) . \delta\left(C_{3 a, 7 a}\right)$ for sodium indenyl $=130.7$ ppm. ${ }^{2}$

${ }^{2}$ Gamasa, M. P.; Gimeno, J.; Gonzalez-Bernardo, C.; Martin-Vaca, B. M. Organometallics 1996, 15, 302. 
NMR spectra for complexes 3c, 6, 7, and 9:

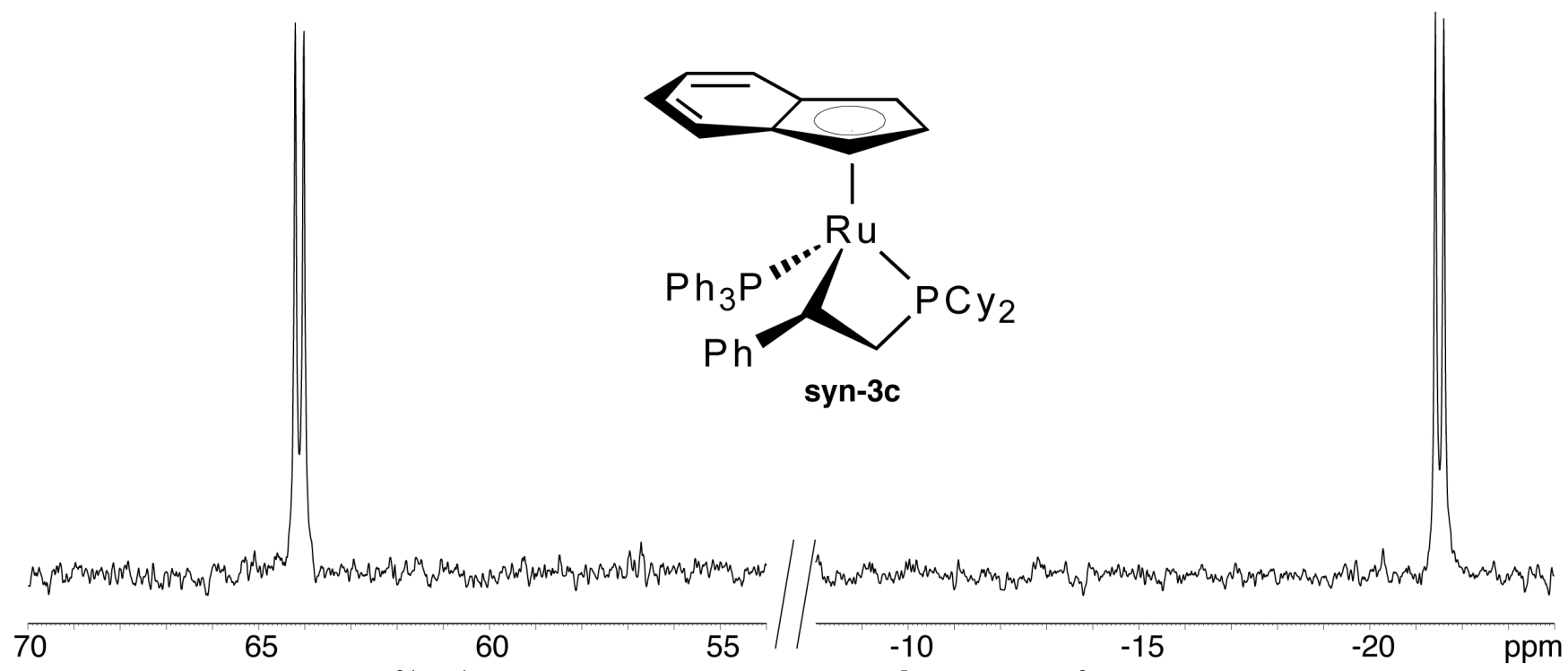

Figure S4. 202.46 MHz ${ }^{31} \mathrm{P}\left\{{ }^{1} \mathrm{H}\right\}$ NMR spectrum of $\mathrm{Ru}\left(\eta^{5}\right.$-indenyl $)\left(\mathrm{k}^{2}-\mathrm{PhCH}_{2} \mathrm{CHPCy}_{2}\right)\left(\mathrm{PPh}_{3}\right)(3 \mathrm{c})$ in $d_{6}$-benzene.

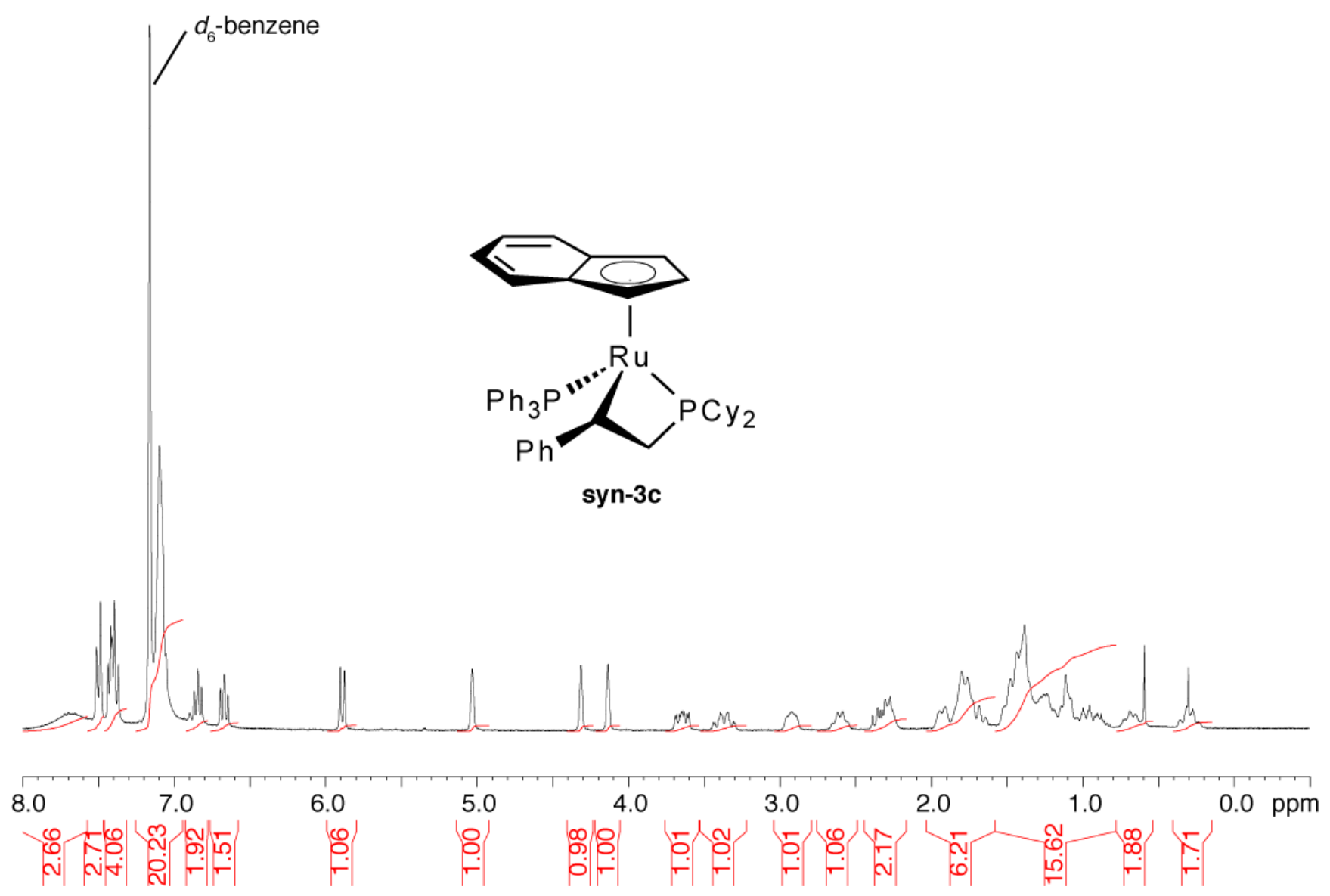

Figure S5. $500.13 \mathrm{MHz}{ }^{1} \mathrm{H}$ NMR spectrum of $\mathrm{Ru}\left(\eta^{5}\right.$-indenyl $)\left(\mathrm{k}^{2}-\mathrm{PhCH}_{2} \mathrm{CHPCy}_{2}\right)\left(\mathrm{PPh}_{3}\right)(3 \mathrm{c})$ in $d_{6^{-}}$ benzene. 

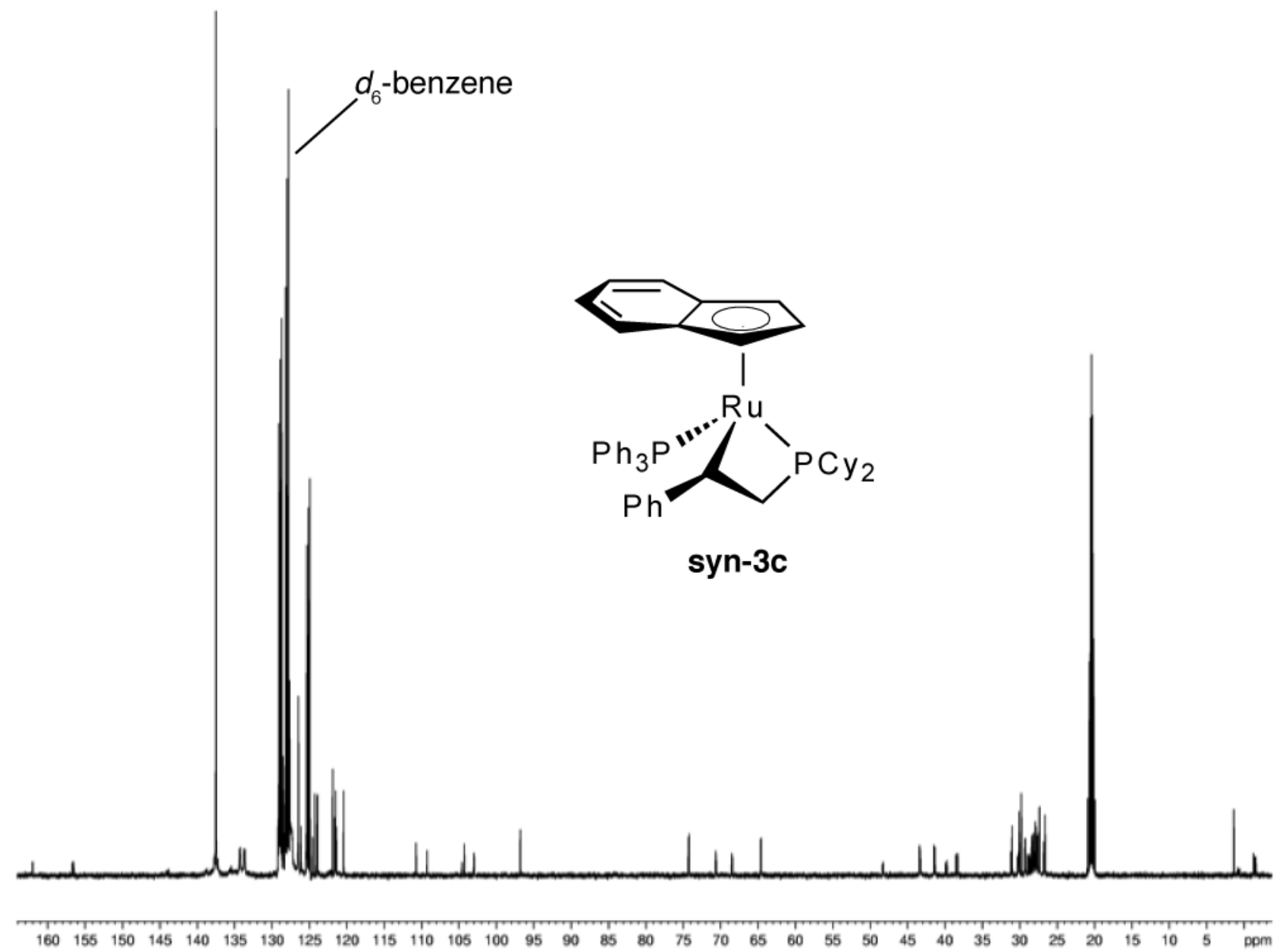

Figure S6. $125.77 \mathrm{MHz}{ }^{13} \mathrm{C}\left\{{ }^{1} \mathrm{H}\right\} \mathrm{NMR}$ spectrum of $\mathrm{Ru}\left(\eta^{5}\right.$-indenyl $)\left(\mathrm{k}^{2}-\mathrm{PhCH}_{2} \mathrm{CHPCy}_{2}\right)\left(\mathrm{PPh}_{3}\right)(\mathbf{3 c})$ in $d_{6}$-benzene. 


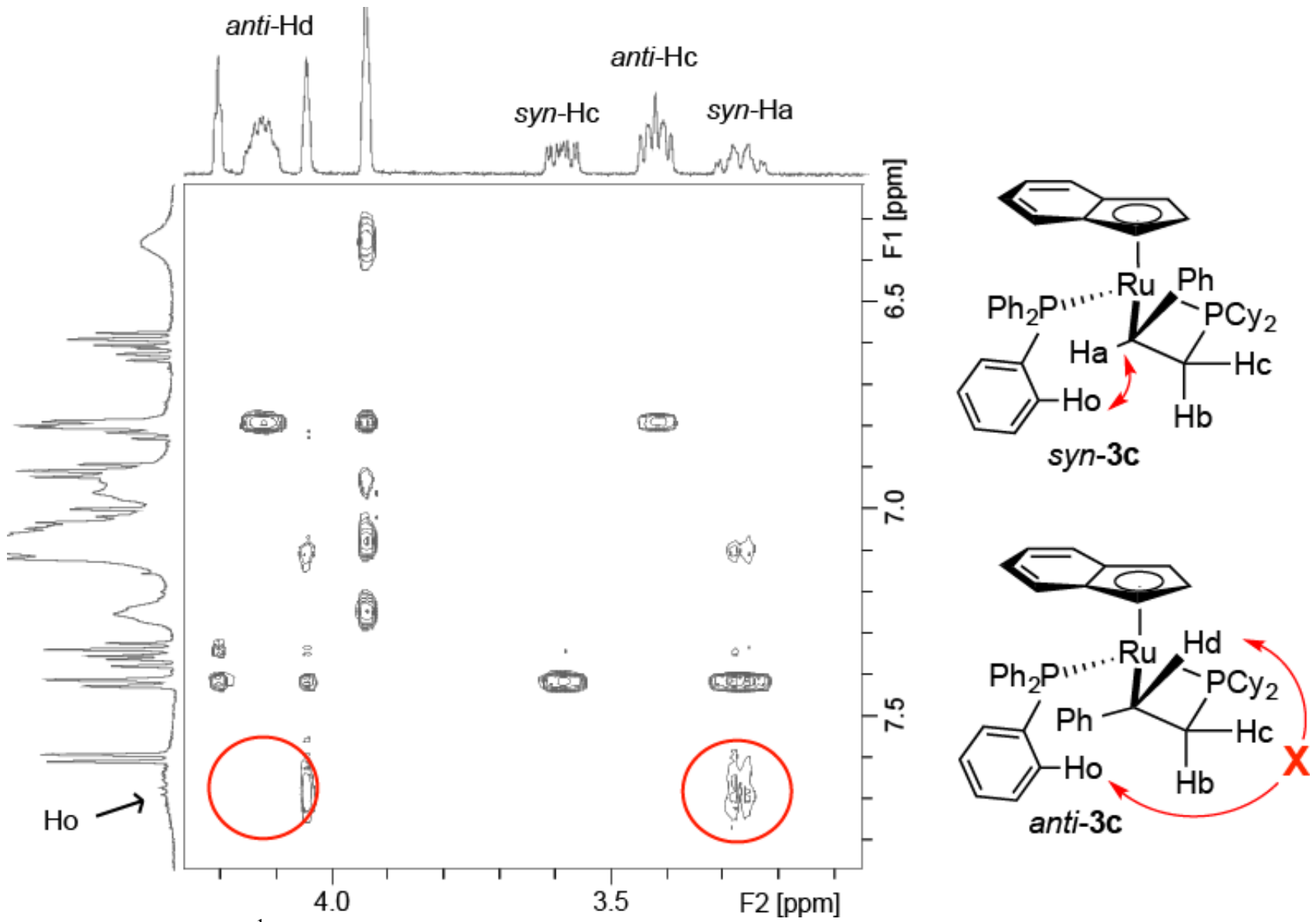

Figure S7. Partial ${ }^{1} \mathrm{H}$ NOESY NMR spectrum of a mixture of $s y n$ - and anti-3c shows stereochemical determination of $s y n-3 \mathbf{c}$ from Ha « Ho correlation. (500.13 MHz, $d_{6}$-benzene) 


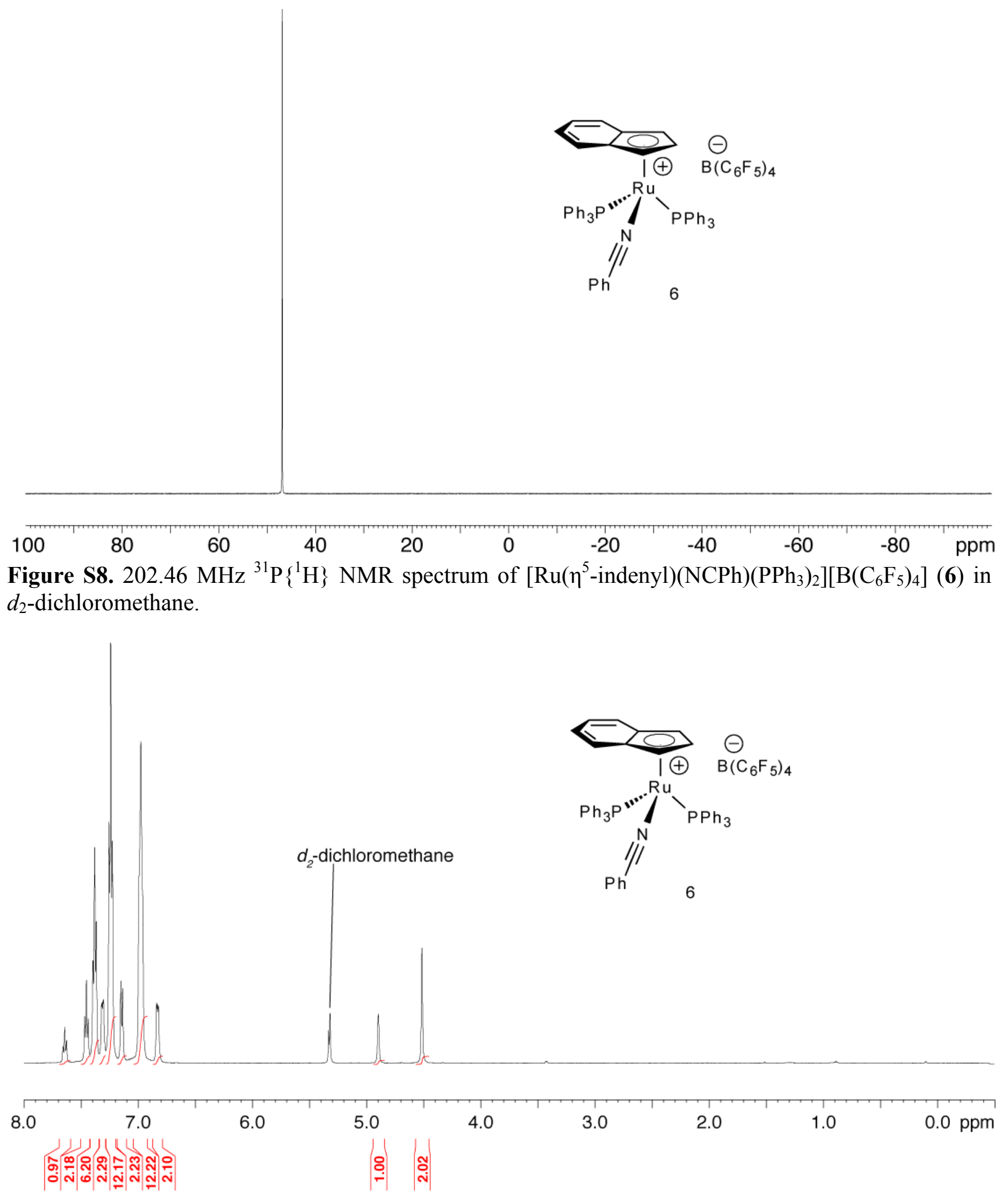

Figure S9. $500.13 \mathrm{MHz}{ }^{1} \mathrm{H} \mathrm{NMR}$ spectrum of $\left[\mathrm{Ru}\left(\eta^{5}\right.\right.$-indenyl $\left.)(\mathrm{NCPh})\left(\mathrm{PPh}_{3}\right)_{2}\right]\left[\mathrm{B}\left(\mathrm{C}_{6} \mathrm{~F}_{5}\right)_{4}\right](\mathbf{6})$ in $d_{2^{-}}$ dichloromethane. 

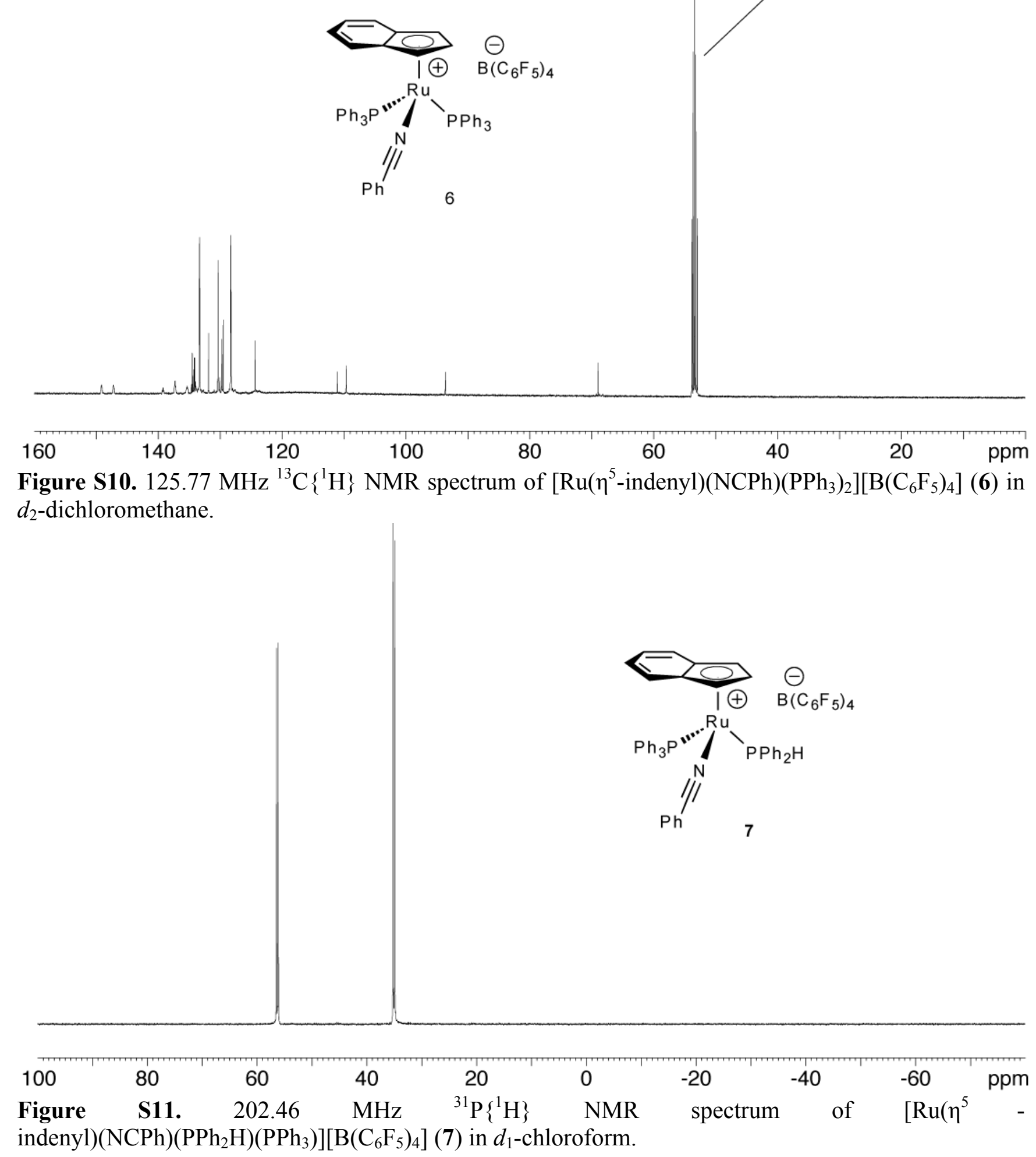

$d_{2}$-dichloromethane 


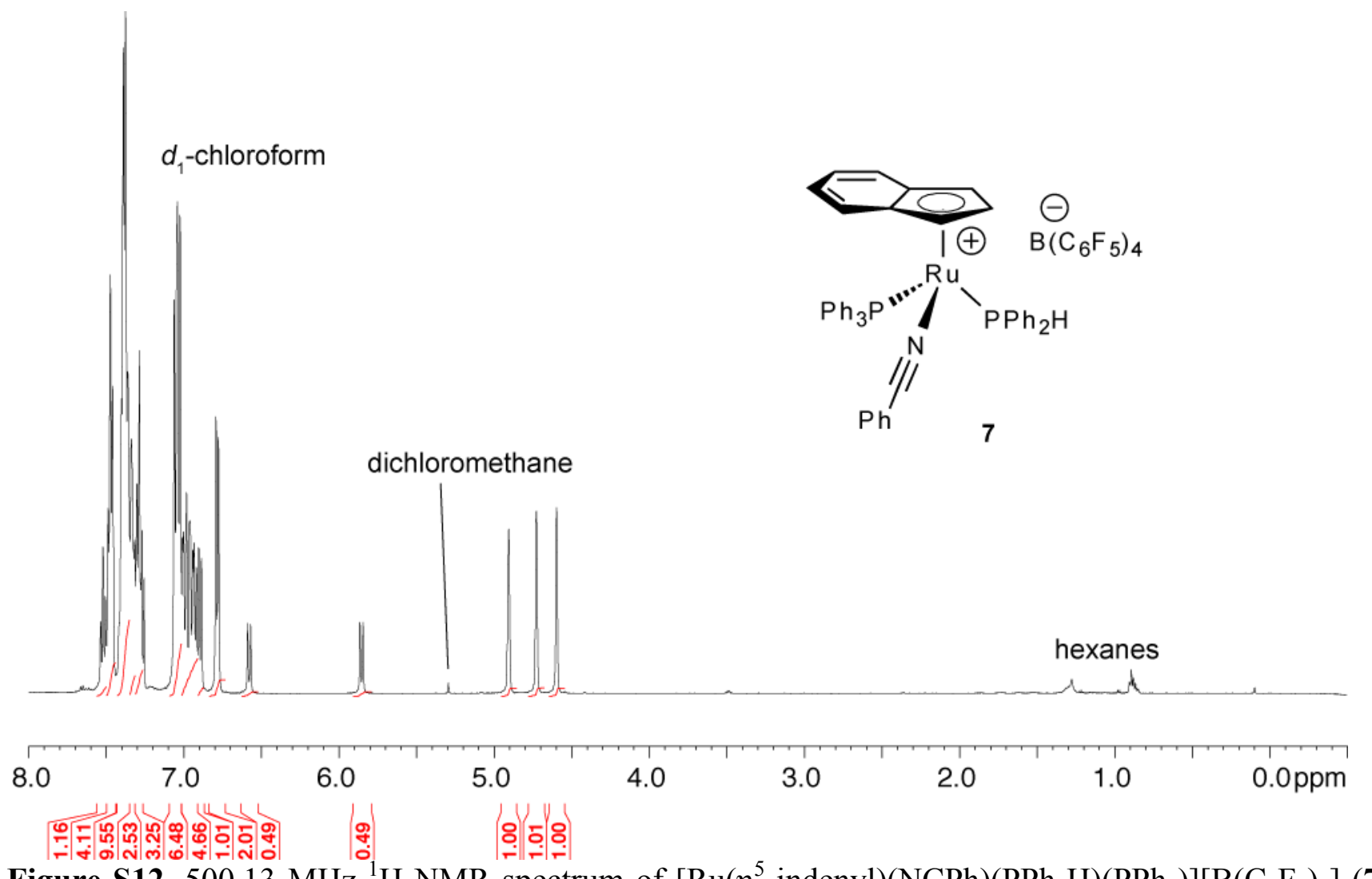

Figure S12. $500.13 \mathrm{MHz}{ }^{1} \mathrm{H}$ NMR spectrum of $\left[\mathrm{Ru}\left(\eta^{5}\right.\right.$-indenyl $\left.)(\mathrm{NCPh})\left(\mathrm{PPh}_{2} \mathrm{H}\right)\left(\mathrm{PPh}_{3}\right)\right]\left[\mathrm{B}\left(\mathrm{C}_{6} \mathrm{~F}_{5}\right)_{4}\right](7)$ in $d_{1}$-chloroform.

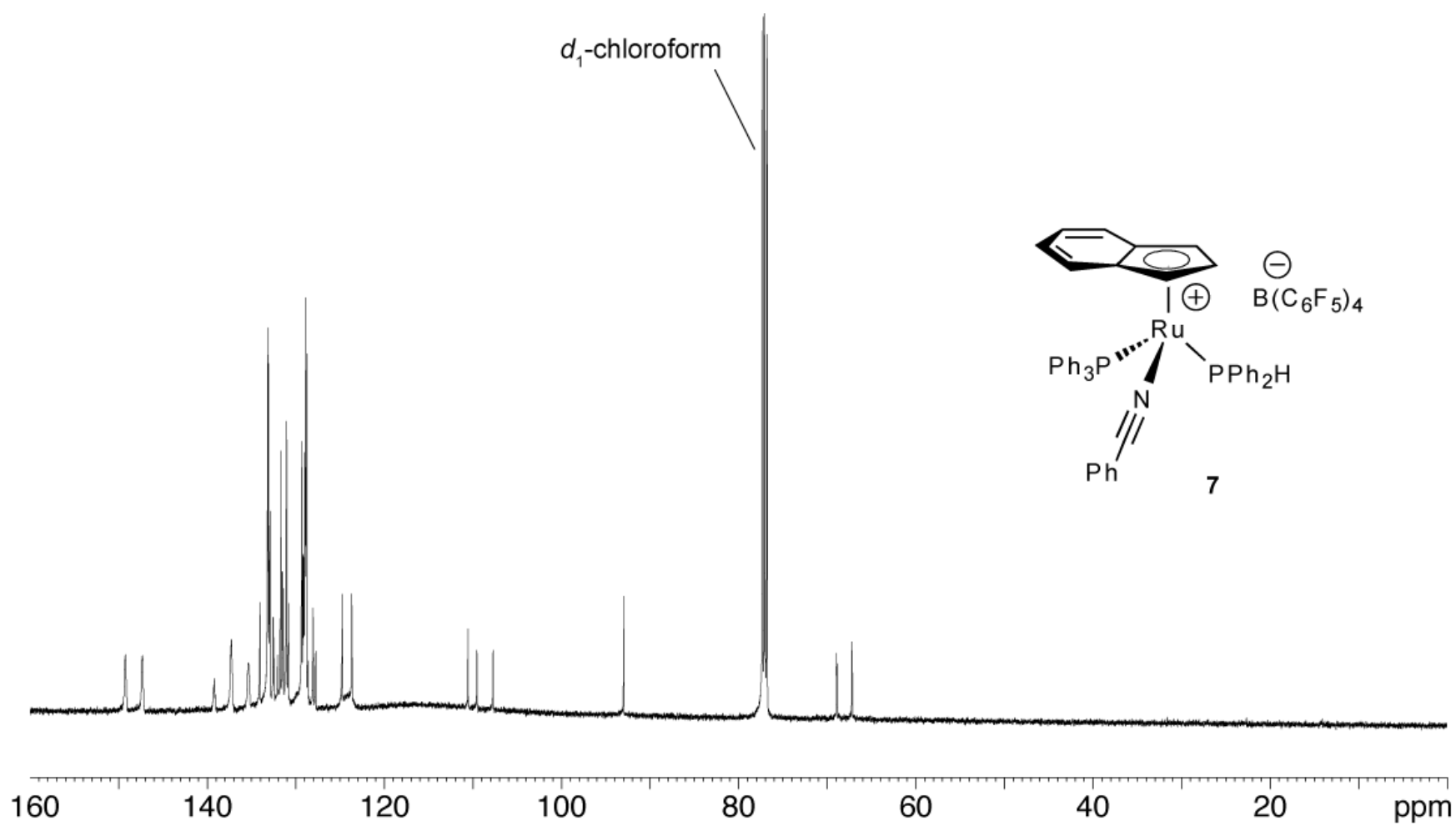

Figure S13. $125.77 \mathrm{MHz}{ }^{13} \mathrm{C}\left\{{ }^{1} \mathrm{H}\right\} \mathrm{NMR}$ spectrum of $\left[\mathrm{Ru}\left(\eta^{5}\right.\right.$-indenyl $\left.)(\mathrm{NCPh})\left(\mathrm{PPh}_{2} \mathrm{H}\right)\left(\mathrm{PPh}_{3}\right)\right]\left[\mathrm{B}\left(\mathrm{C}_{6} \mathrm{~F}_{5}\right)_{4}\right]$ (7) in $d_{1}$-chloroform. 


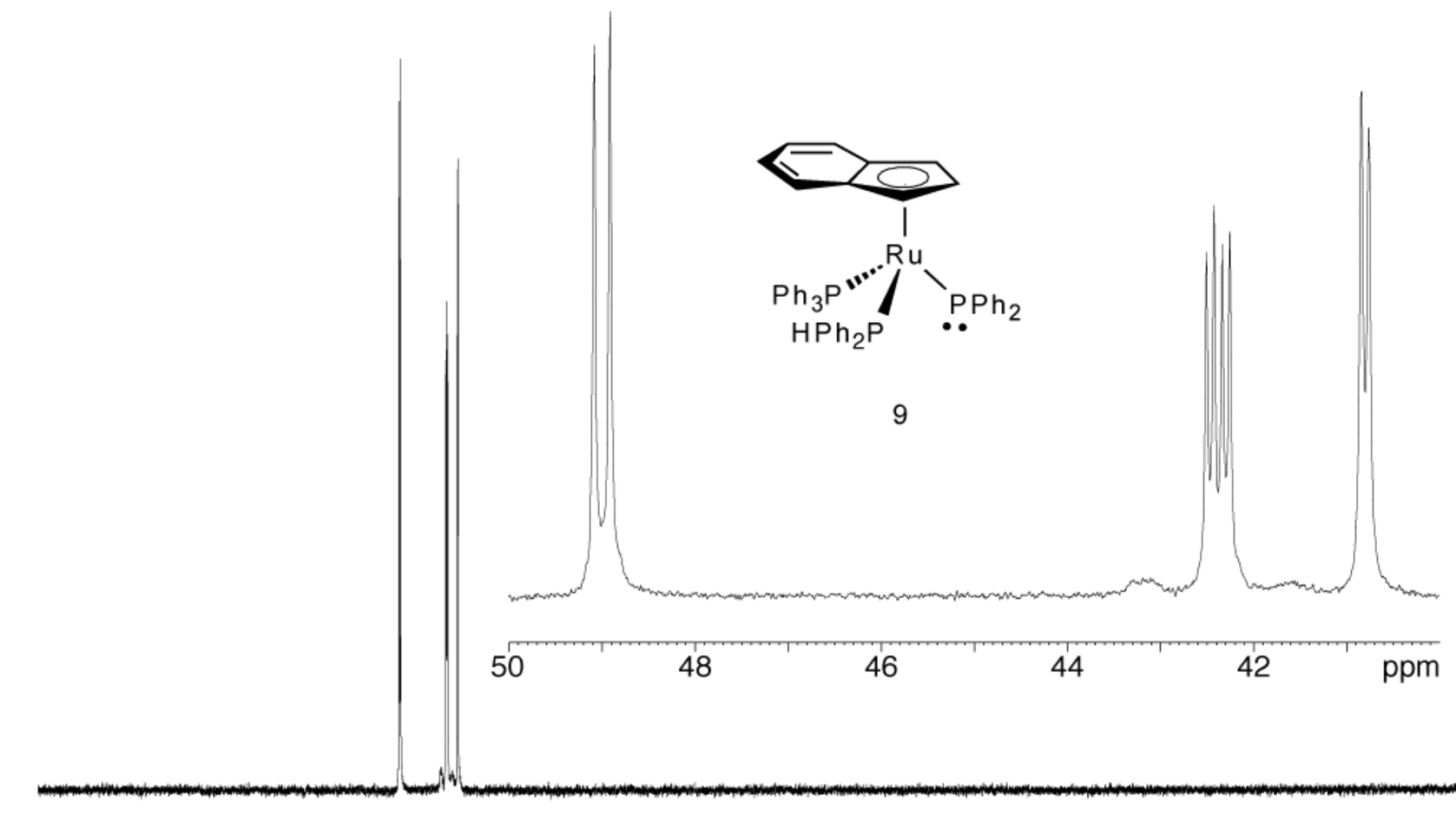

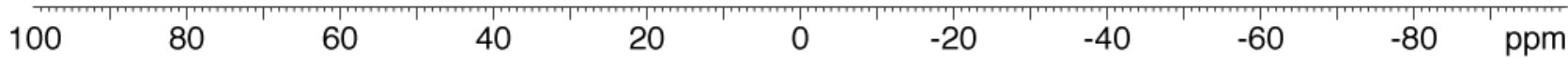

Figure S14. 202.46 MHz ${ }^{31} \mathrm{P}\left\{{ }^{1} \mathrm{H}\right\}$ NMR spectrum of $\left[\mathrm{Ru}\left(\eta^{5}\right.\right.$-indenyl $\left.)\left(\mathrm{PPh}_{2}\right)\left(\mathrm{PPh}_{2} \mathrm{H}\right)\left(\mathrm{PPh}_{3}\right)\right](9)$ in $d_{8^{-}}$ THF.

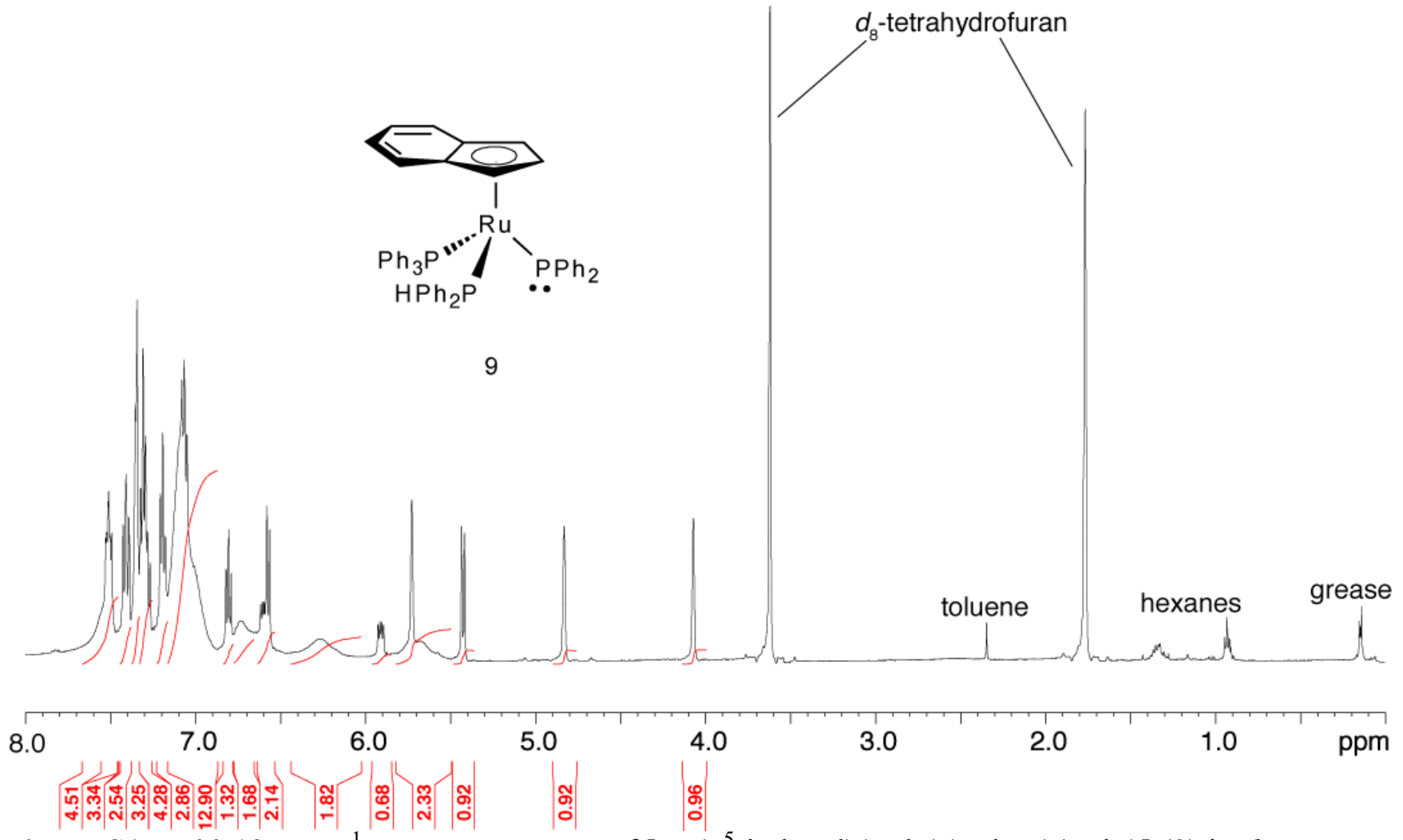

Figure S15. $500.13 \mathrm{MHz}{ }^{1} \mathrm{H}$ NMR spectrum of $\left[\mathrm{Ru}\left(\eta^{5}\right.\right.$-indenyl $\left.)\left(\mathrm{PPh}_{2}\right)\left(\mathrm{PPh}_{2} \mathrm{H}\right)\left(\mathrm{PPh}_{3}\right)\right](9)$ in $d_{8}$-THF. 


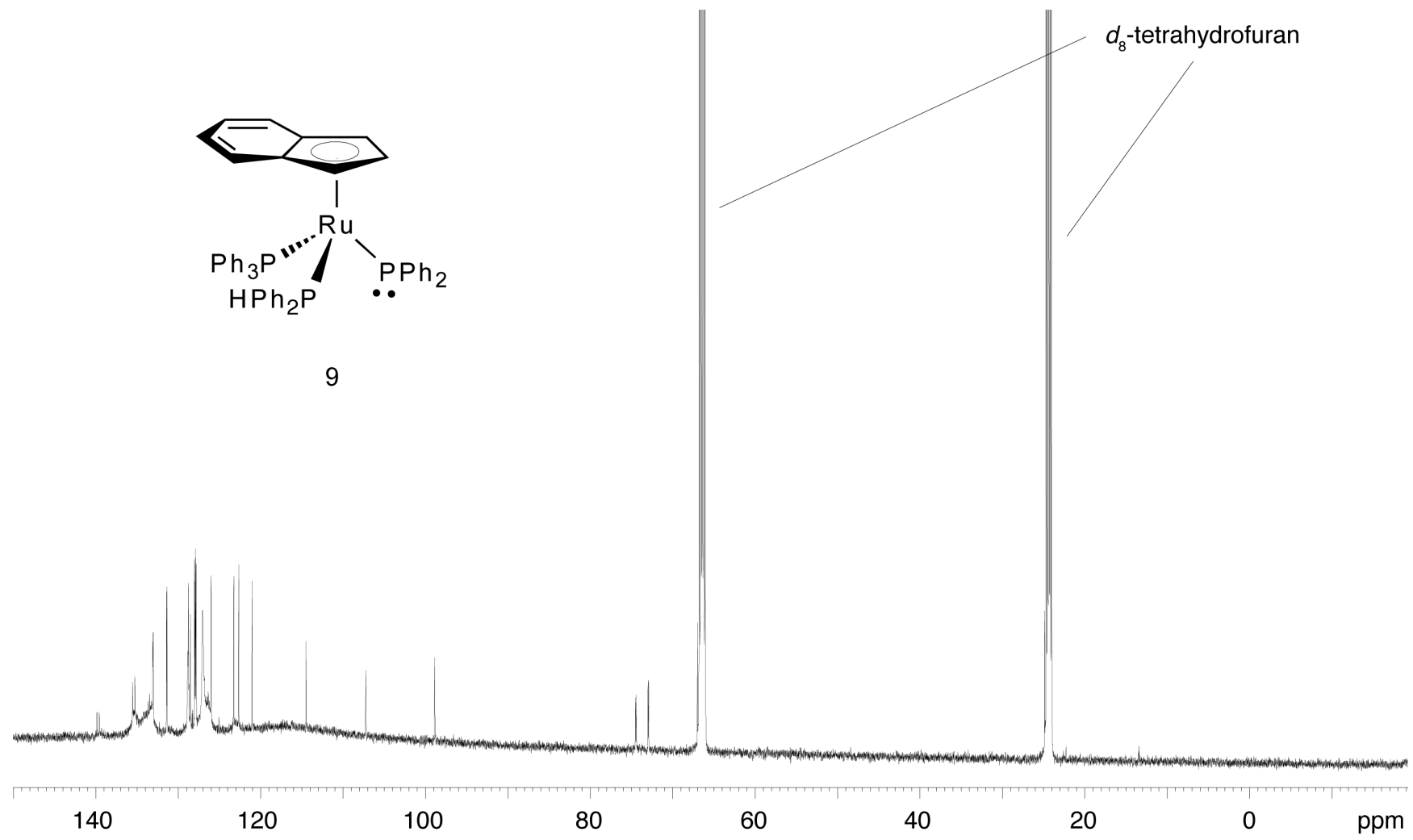

Figure S16. $125.77 \mathrm{MHz}{ }^{13} \mathrm{C}\left\{{ }^{1} \mathrm{H}\right\}$ NMR spectrum of $\left[\mathrm{Ru}\left(\eta^{5}\right.\right.$-indenyl $\left.)\left(\mathrm{PPh}_{2}\right)\left(\mathrm{PPh}_{2} \mathrm{H}\right)\left(\mathrm{PPh}_{3}\right)\right](9)$ in $d_{8^{-}}$ THF. 
Representative $145.78 \mathrm{MHz}{ }^{31} \mathrm{P}\left\{{ }^{1} \mathrm{H}\right\}$ NMR spectra of catalyst screening reactions, and associated control reactions, carried out in $3: 1 \mathrm{THF} / d_{6}$-benzene:

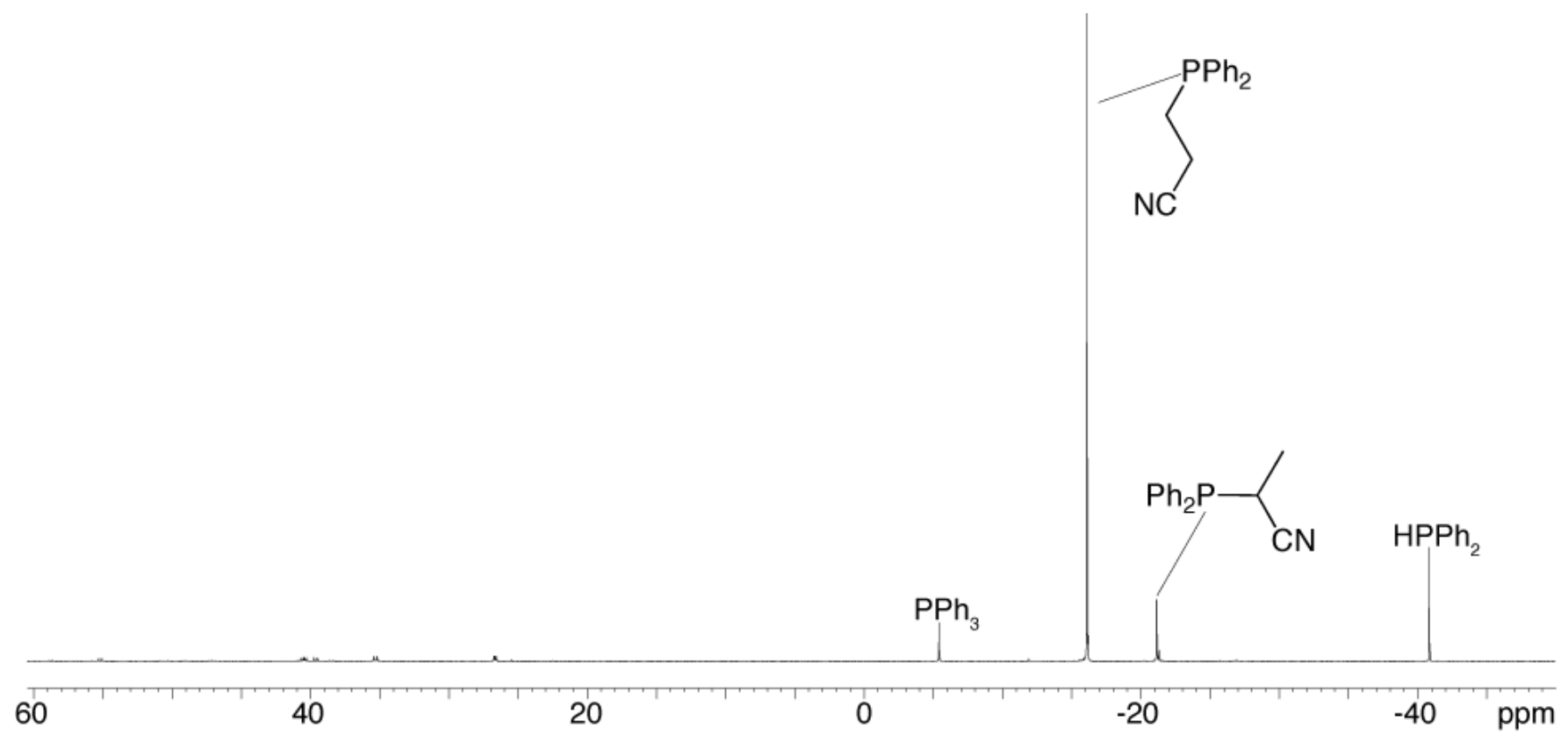

Figure S17. Mixture of acrylonitrile, $\mathrm{HPPh}_{2}, 10 \mathrm{~mol} \% \mathrm{8}$, and $10 \mathrm{~mol} \% \mathrm{DBU}$ after $24 \mathrm{~h}$ (entry 3, Table 2). Entries 1, 2, 4, 7, 10, and 11 produced similar spectra.

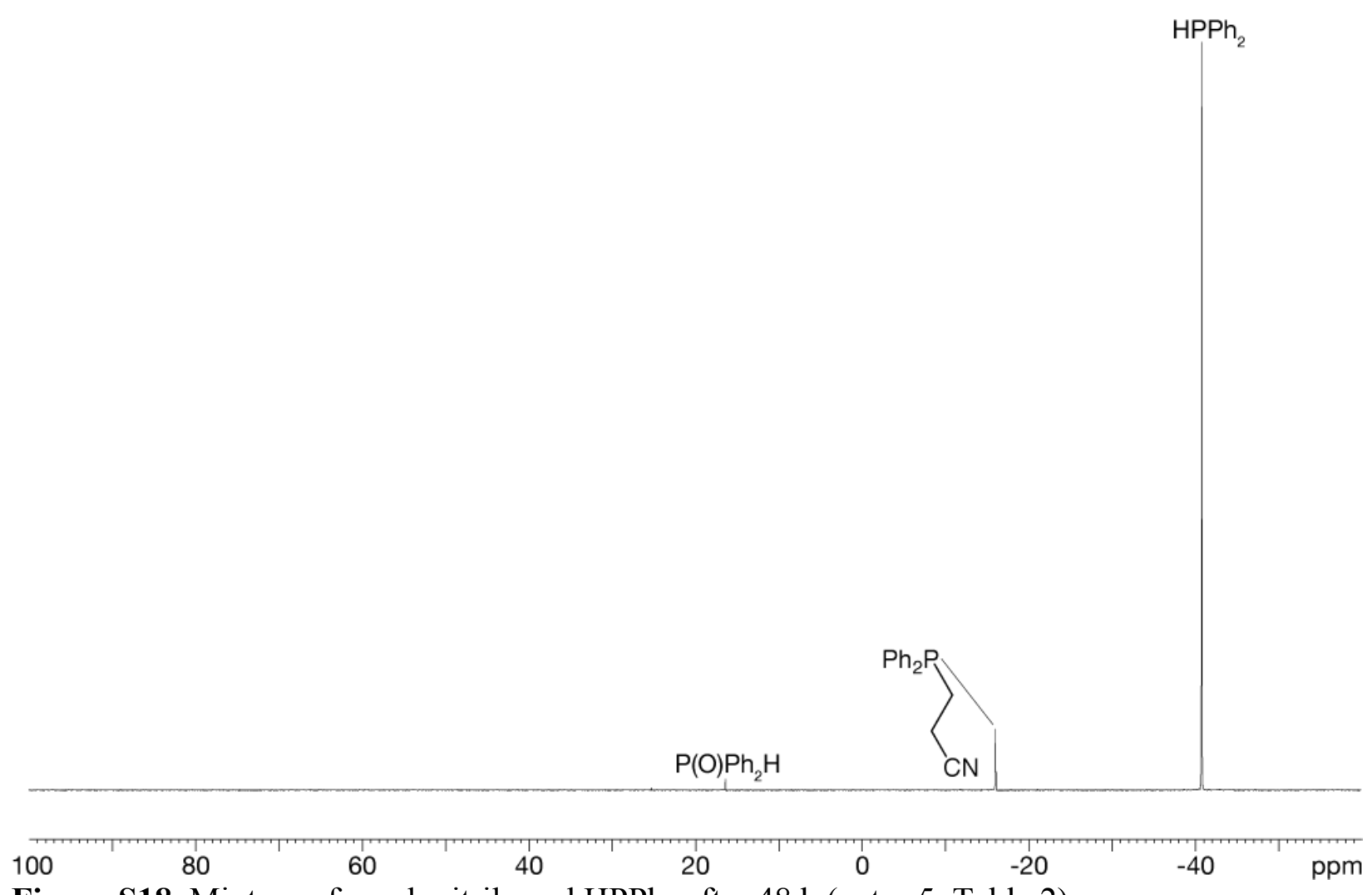

Figure S18. Mixture of acrylonitrile and $\mathrm{HPPh}_{2}$ after $48 \mathrm{~h}$ (entry 5, Table 2) 


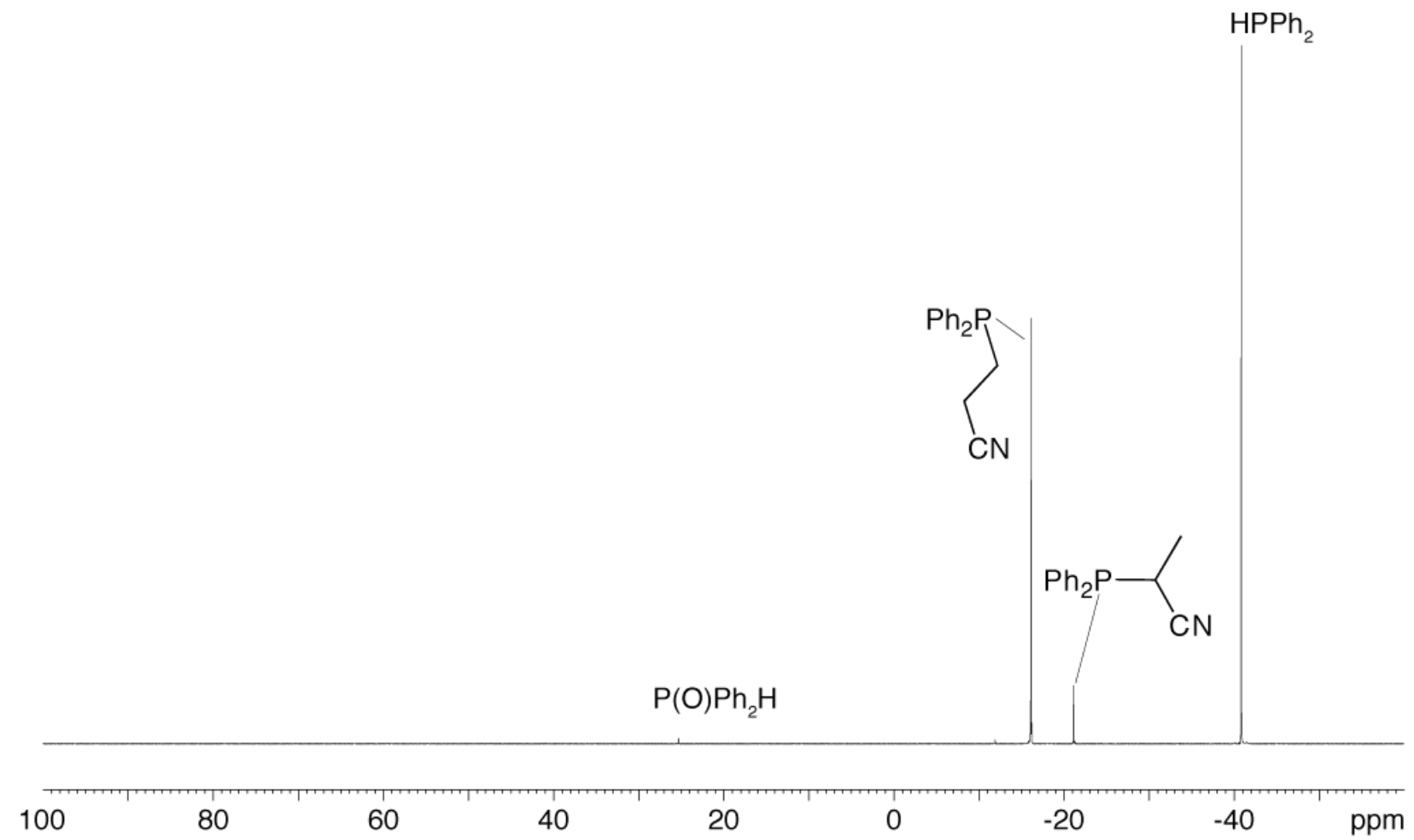

Figure S19. Mixture of acrylonitrile, $\mathrm{HPPh}_{2}$, and $10 \mathrm{~mol} \% \mathrm{DBU}$ after $48 \mathrm{~h}$ (entry 6, Table 2).

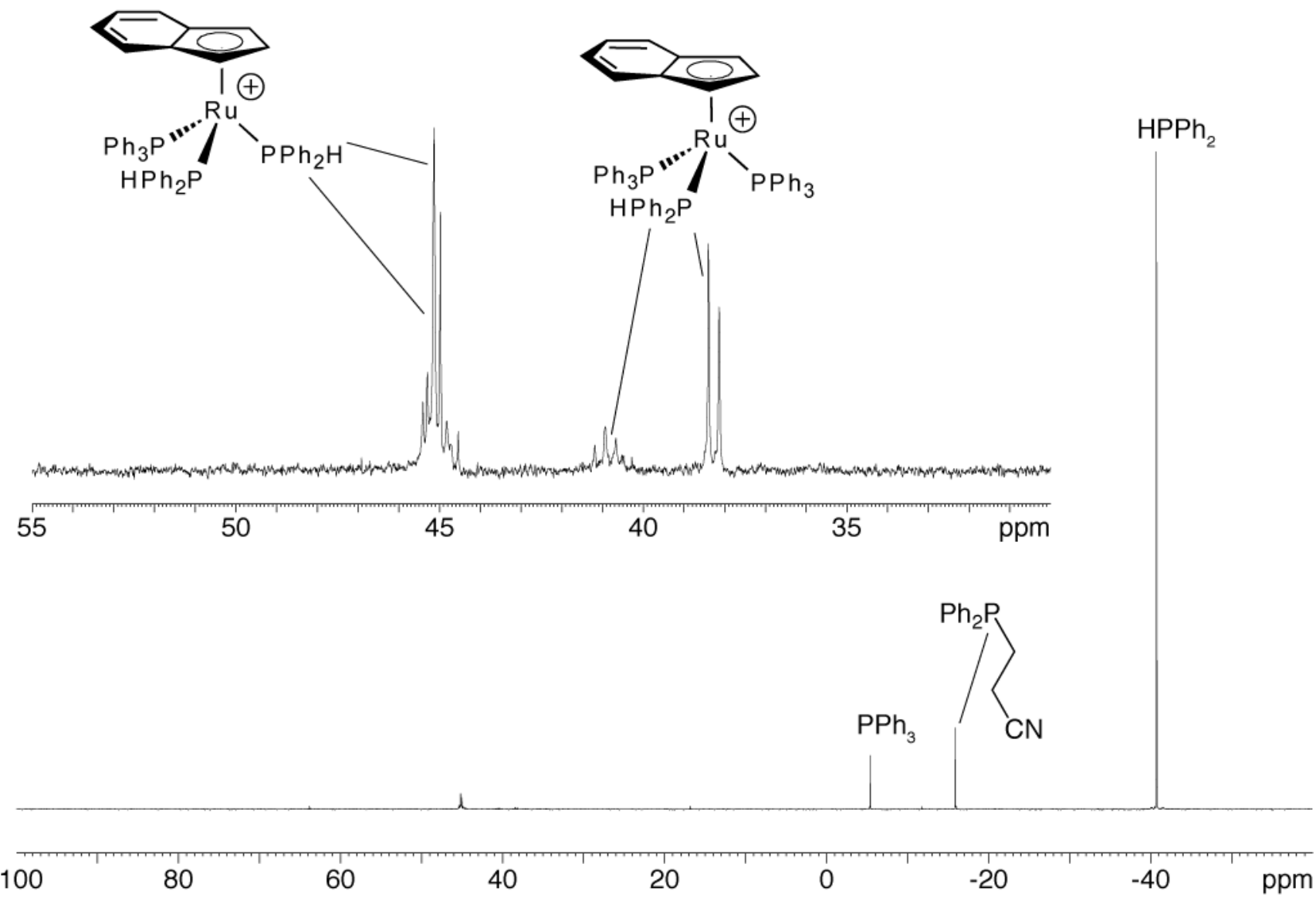

Figure S20. Mixture of acrylonitrile, $\mathrm{HPPh}_{2}$, and $10 \mathrm{~mol} \%$ 6, after $48 \mathrm{~h}$ (entry 8, Table 2). Tentative assignments for substitution products are indicated. 


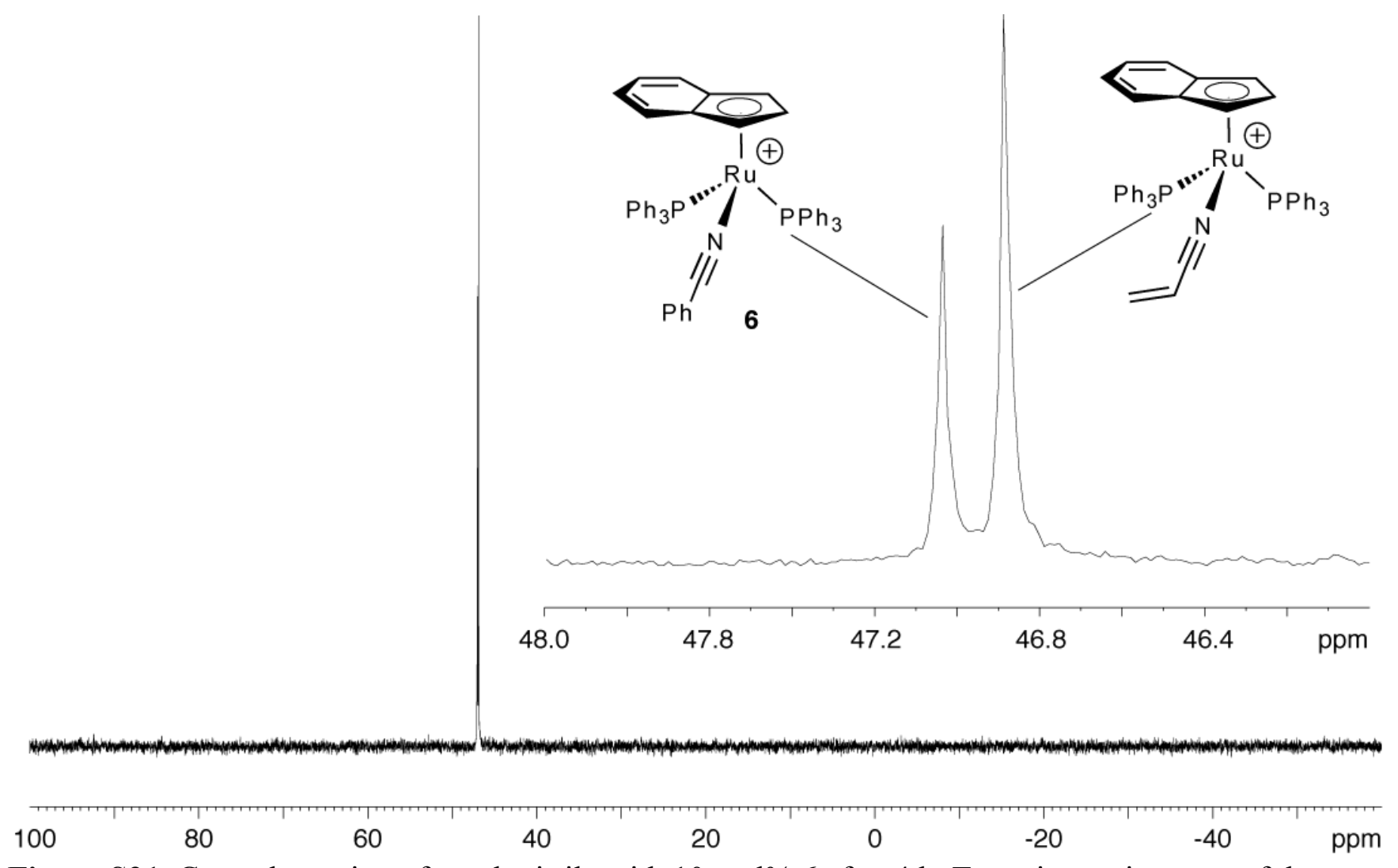

Figure S21. Control reaction of acrylonitrile with $10 \mathrm{~mol} \% 6$ after 4 h. Tentative assignment of the substitution product $\left[\mathrm{Ru}\left(\eta^{5}\right.\right.$-indenyl $\left.)\left(\mathrm{NCCHCH}_{2}\right)\left(\mathrm{PPh}_{3}\right)_{2}\right]\left[\mathrm{B}\left(\mathrm{C}_{6} \mathrm{~F}_{5}\right)_{4}\right]$ in indicated.
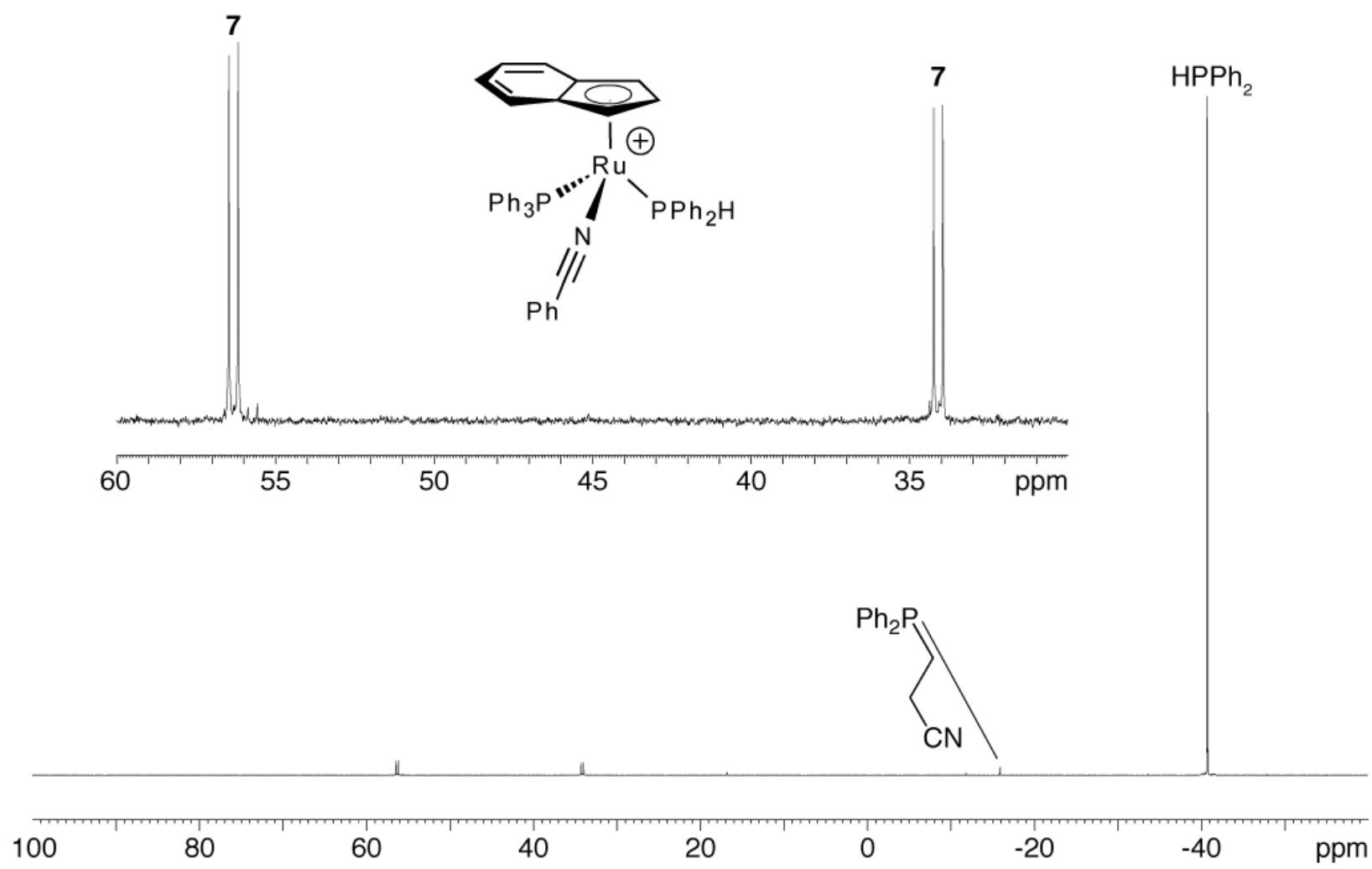

Figure S22. Mixture of acrylonitrile, $\mathrm{HPPh}_{2}$, and $10 \mathrm{~mol} \% 7$ after $48 \mathrm{~h}$ (entry 9, Table 2). 


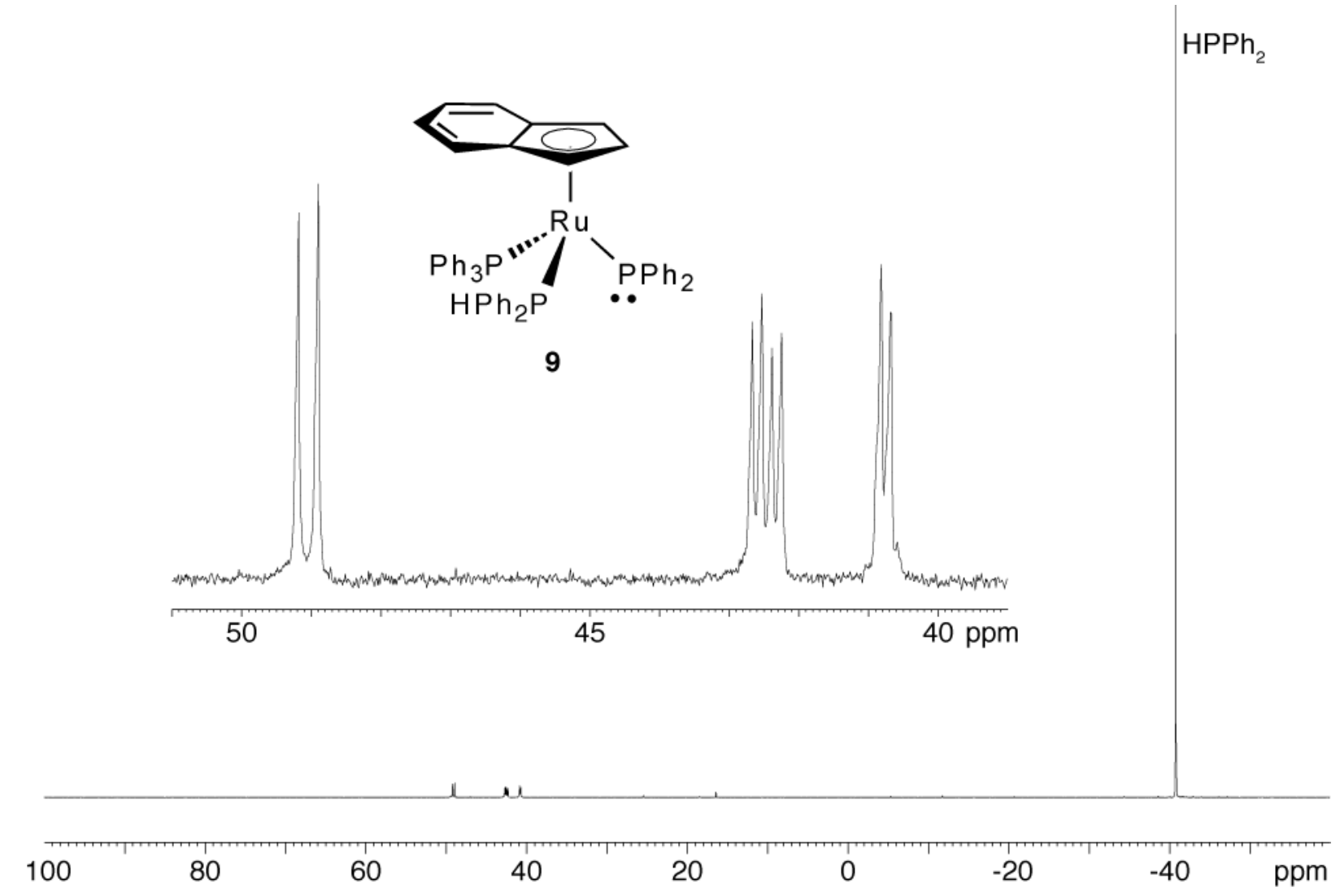

$\mathrm{HPPh}_{2}$

Figure S23. Mixture of 1-hexene, $\mathrm{HPPh}_{2}$, and $10 \mathrm{~mol} \% 8$ after 48 h (entry 13, Table 2). Entry 12 produced a similar spectrum.

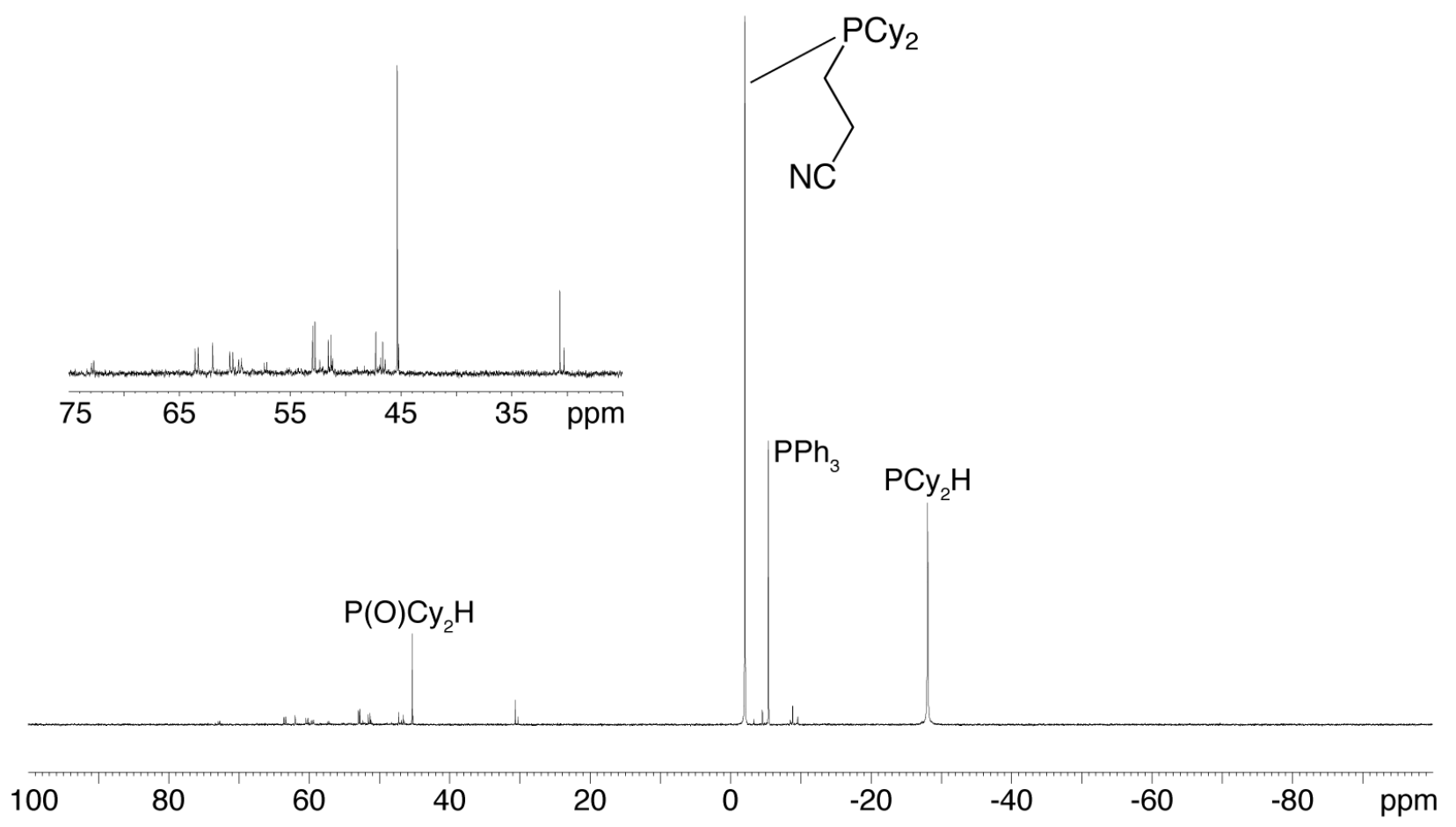

Figure S24. Mixture of acrylonitrile, $\mathrm{HPCy}_{2}, 10 \mathrm{~mol} \%$ 6, and $10 \mathrm{~mol} \% \mathrm{DBU}$ after $48 \mathrm{~h}$ (entry 14, Table 2). 


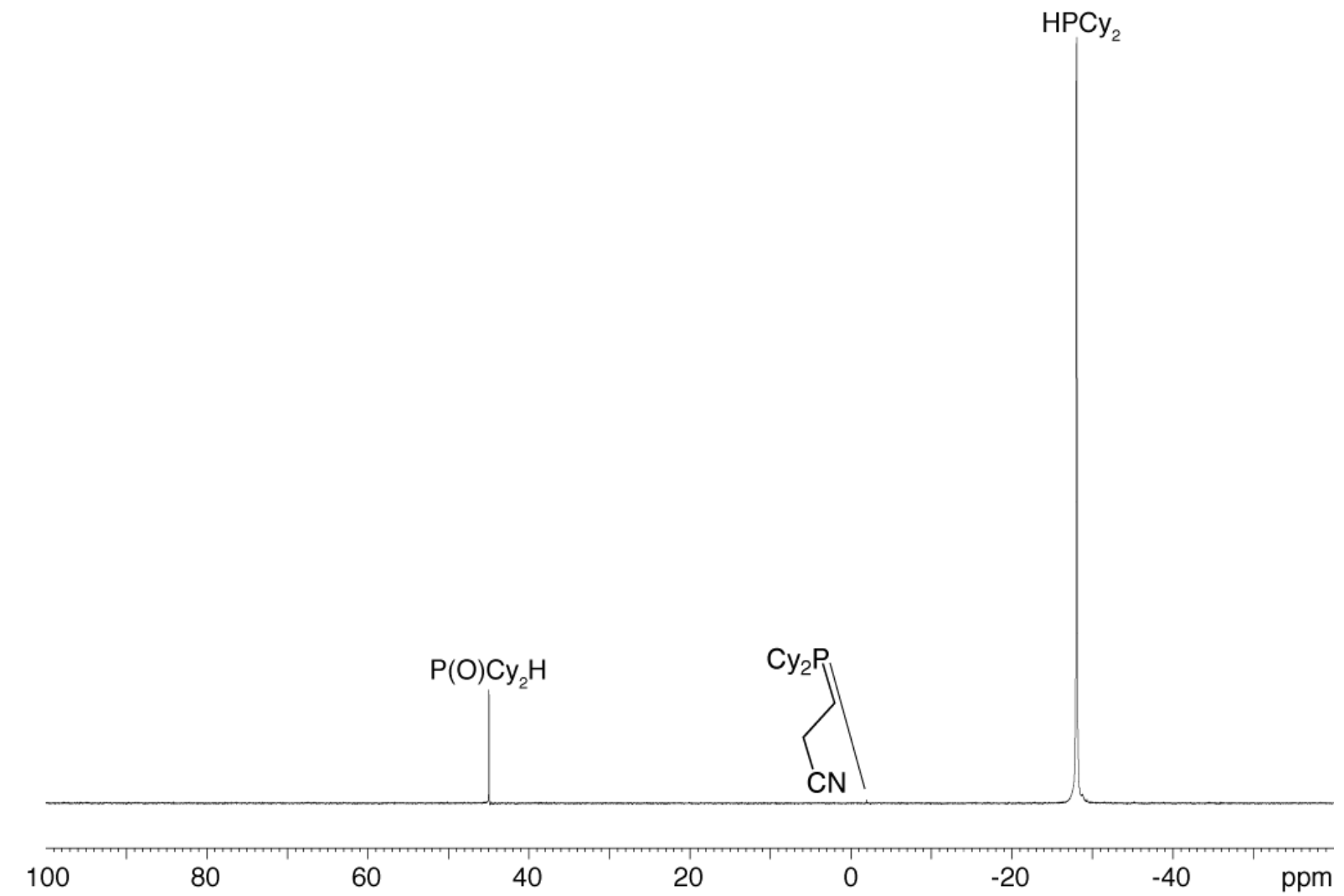

Figure S25. Mixture of acrylonitrile and $\mathrm{HPCy}_{2}$ after $48 \mathrm{~h}$ (entry 15, Table 2). Entry 16 produced a similar spectrum.
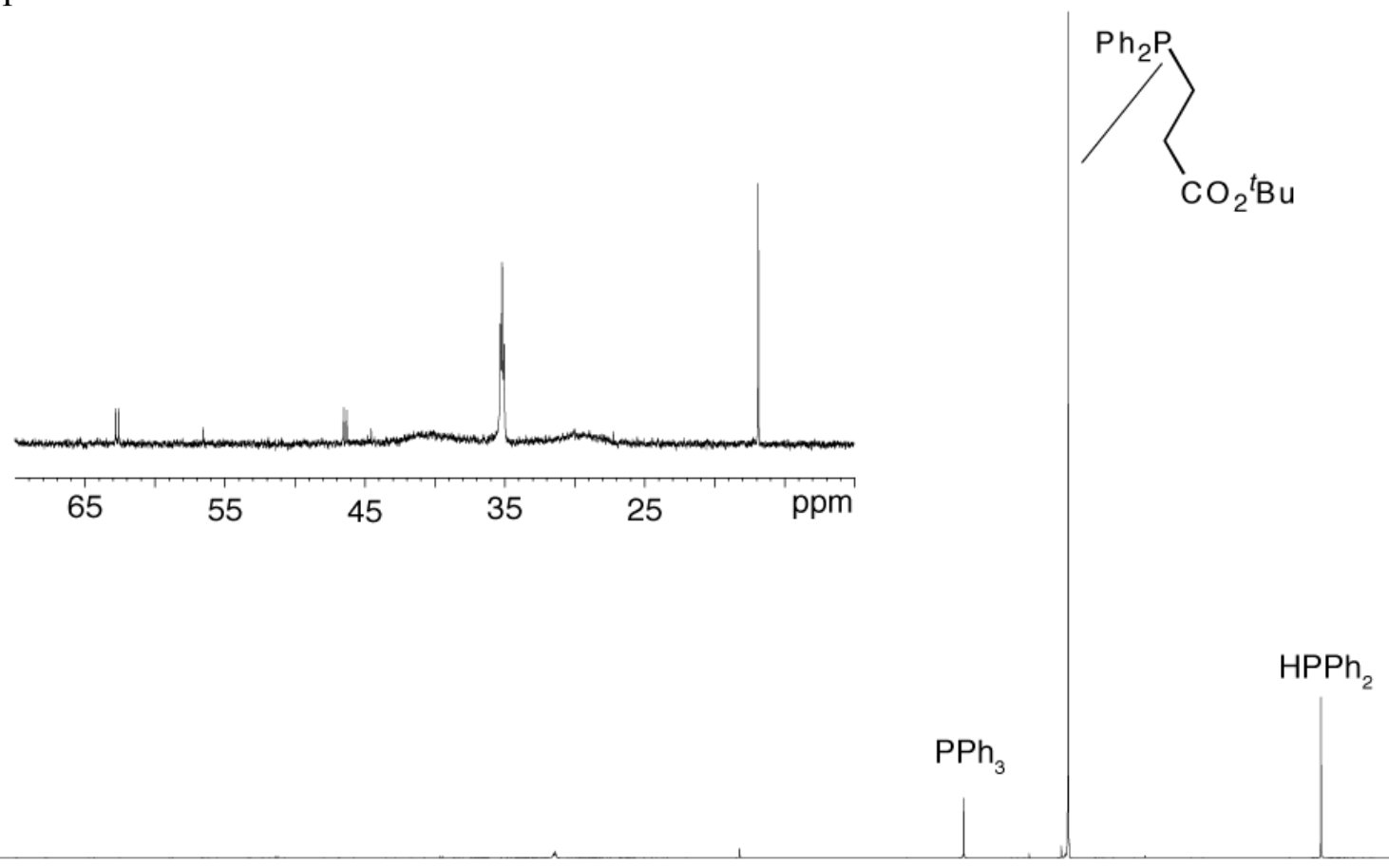

100

80

60

40

20

$-20$

$-40$

ppm

Figure S26. Mixture of tert-butyl acrylate, $\mathrm{HPPh}_{2}$, and $10 \mathrm{~mol} \% \mathbf{8}$, after 24 h (entry 14, Table 2). 


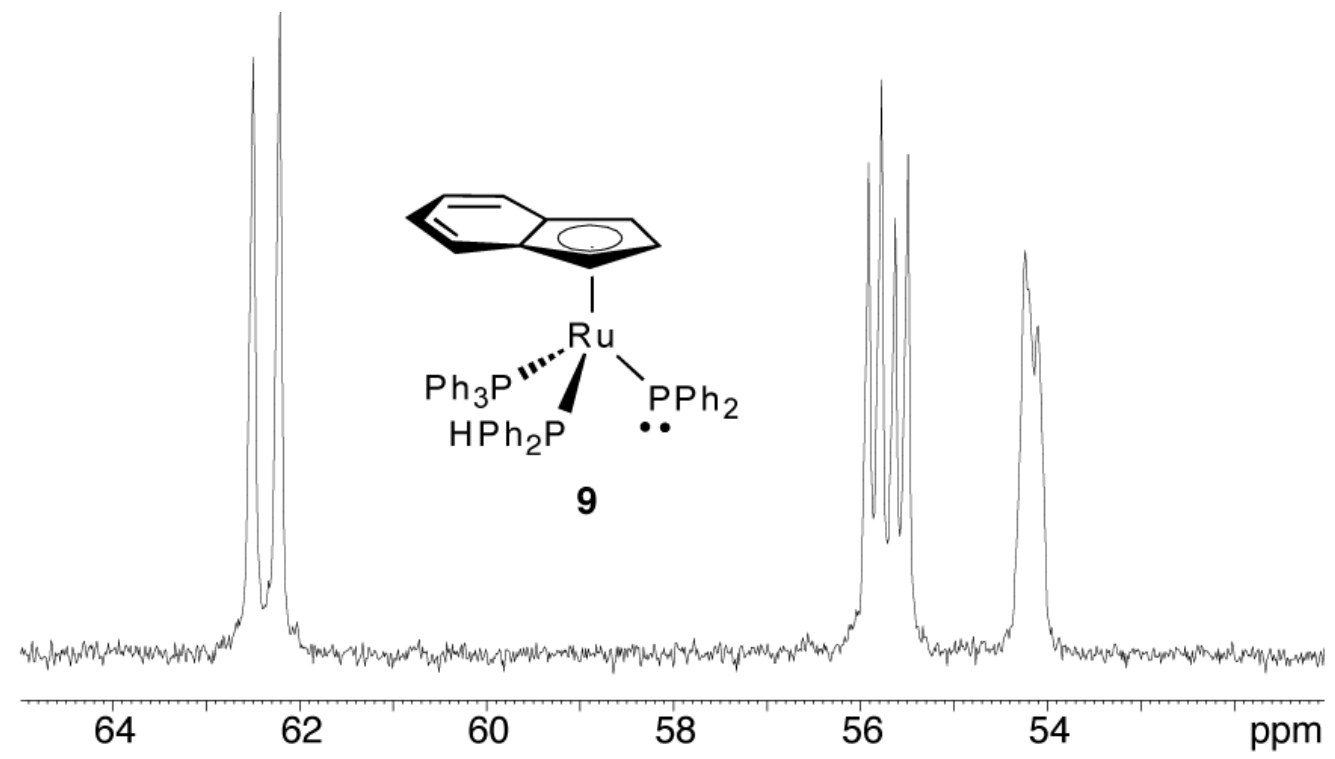

$\mathrm{HPPh}_{2}$

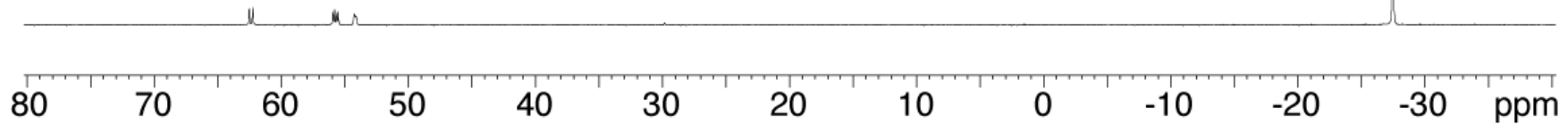

Figure S27. Mixture of 8 and 10 equiv $\mathrm{HPPh}_{2}$. 

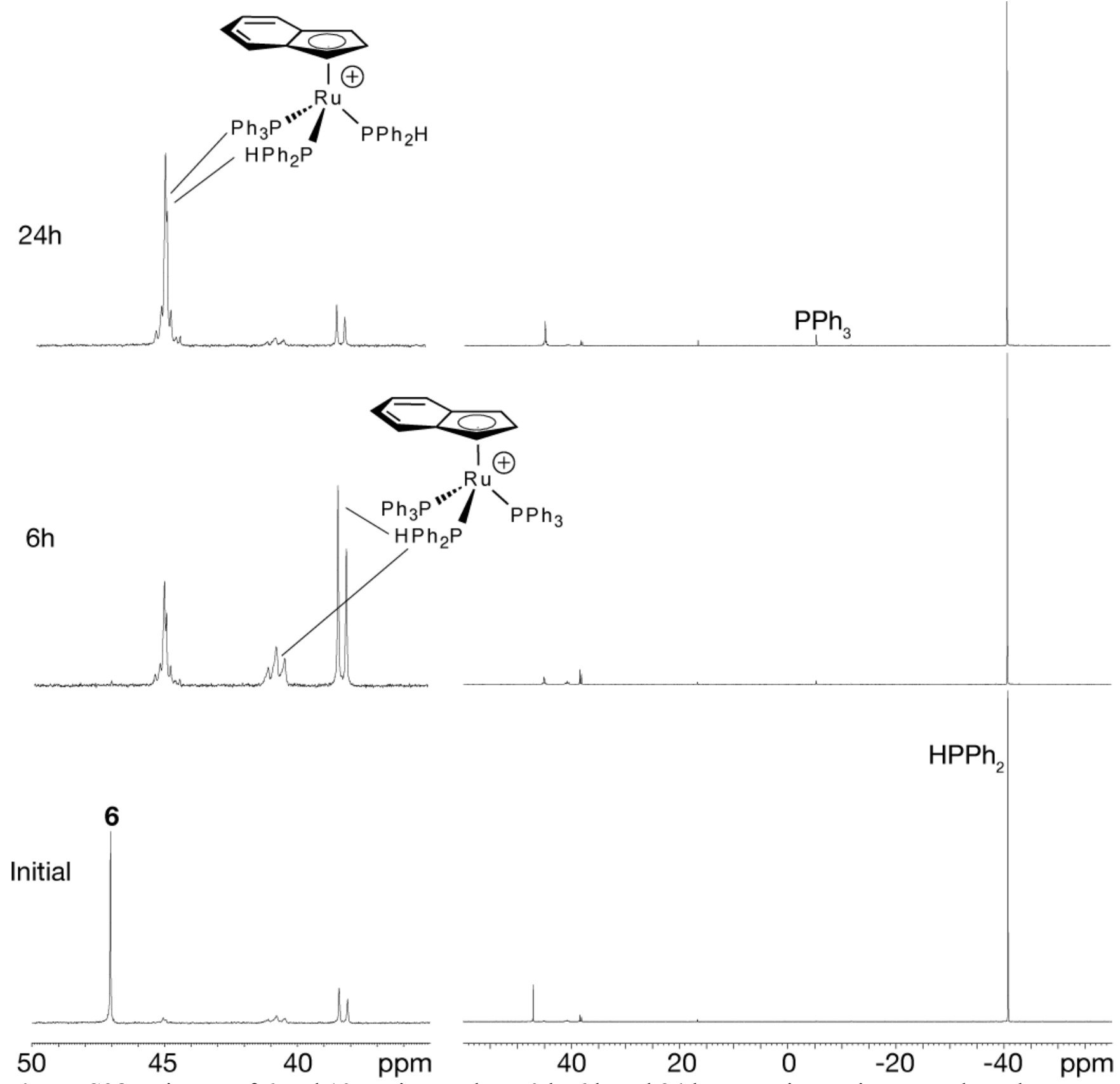

Figure S28. Mixture of 6 and 10 equiv $\mathrm{HPPh}_{2}$ at $0 \mathrm{~h}, 6 \mathrm{~h}$ and $24 \mathrm{~h}$. Tentative assignments have been made for substitution products. 


\section{Expansions of ${ }^{31} \mathrm{P}\left\{{ }^{1} \mathrm{H}\right\}$ NMR spectra showing catalyst resting states for the reactions of acrylonitrile with $\mathrm{HPPh}_{2}$ :}

These catalytic reaction mixtures show signals due to multiple Ru-containing products. Identification of true catalyst resting states, as opposed to irrelevant off-cycle by-products is ongoing, but assignment of some general, recurring structural motifs is possible from these spectra. Figs. S29S34 below have been labelled to indicate signals due to apparently discrete species in the catalytic reaction mixtures. For example, two common Ru-containing species were observed in the catalytic reactions of complexes 6 and 7 (Figs. S29 and S30). These sets of doublets (labelled dark and light blue) with ${ }^{2} J_{\mathrm{PP}}$ of $34 \mathrm{~Hz}$ and $35 \mathrm{~Hz}$, respectively, represent a complex that contains two inequivalent trivalent phosphines. Complex 7 also produced another major product with two inequivalent phosphines, with ${ }^{2} \mathbf{J}_{\mathrm{PP}}$ of $32 \mathrm{~Hz}$ (peaks labelled green in Fig. S30). The neutral complexes 8 and 9 resulted in very similar product mixtures. For reactions where DBU was added (Figs. S31 and S32), the same two sets of doublets that were observed for catalytic mixtures obtained using 6 and 7 were observed as the major species (dark and light blue labels). For reactions of complexes 8 and $\mathbf{9}$ without DBU (Figs. S33 and S34), signals were observed that were consistent with three inequivalent phosphorus nuclei (either $\mathrm{PR}_{3}$ or $\mathrm{PR}_{2}$ ). This was determined by the presence of the resonances 40.5 (dd, $\left.{ }^{2} \mathrm{~J}_{\mathrm{PP}}=34 \mathrm{~Hz}, 23 \mathrm{~Hz}\right), 35.3\left(\mathrm{dd},{ }^{2} \mathrm{~J}_{\mathrm{PP}}=34 \mathrm{~Hz}, 3 \mathrm{~Hz}\right)$ and $26.6\left(\mathrm{dd},{ }^{2} \mathrm{~J}_{\mathrm{PP}}=23 \mathrm{~Hz}, 3 \mathrm{~Hz}\right.$ ) (red dots in Figure S33 and S34). In all cases, approximately 1-3 other minor Ru-species were observed, as well as free $\mathrm{PPh}_{3}$ ( -5 ppm, not shown here).

These complexes probably have the following generic structures, where $\mathrm{PR}_{3}=\mathrm{PPh}_{3}, \mathrm{HPPh}_{2}$, $\mathrm{Ph}_{2} \mathrm{PCH}_{2} \mathrm{CH}_{2} \mathrm{CN}$, or $\mathrm{Ph}_{2} \mathrm{CH}(\mathrm{CN}) \mathrm{CH}_{3}$, and where $\mathrm{X}$ and $\mathrm{L}$ are anionic and neutral (respectively) nonphosphorus donors (to be determined).
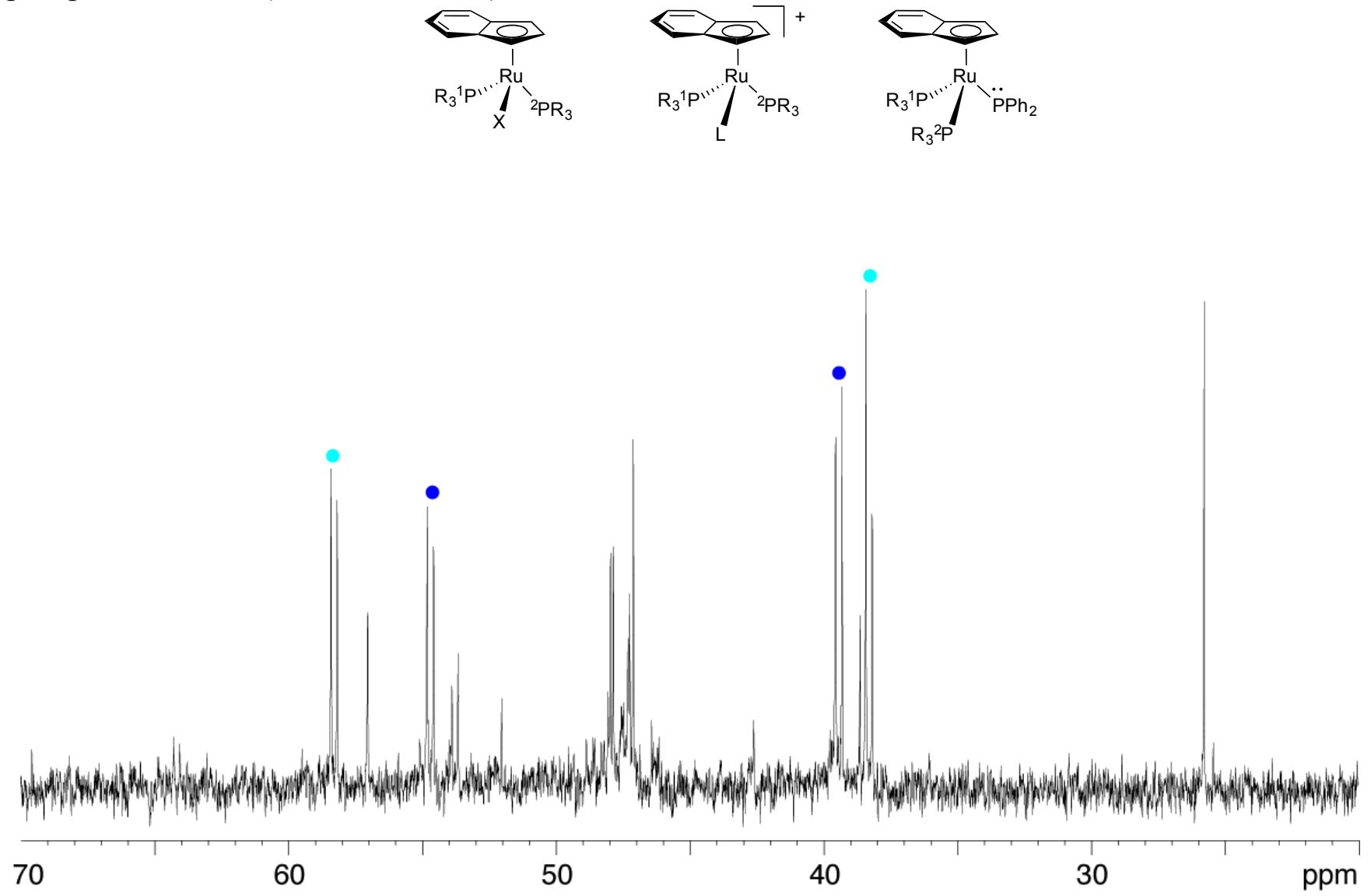

Figure S29. Catalyst resting state resulting from mixture of acrylonitrile, $\mathrm{HPPh}_{2}, 10 \mathrm{~mol} \% \mathbf{6}$, and 10 mol\% DBU after $24 \mathrm{~h}$ (entry 1, Table 2). 


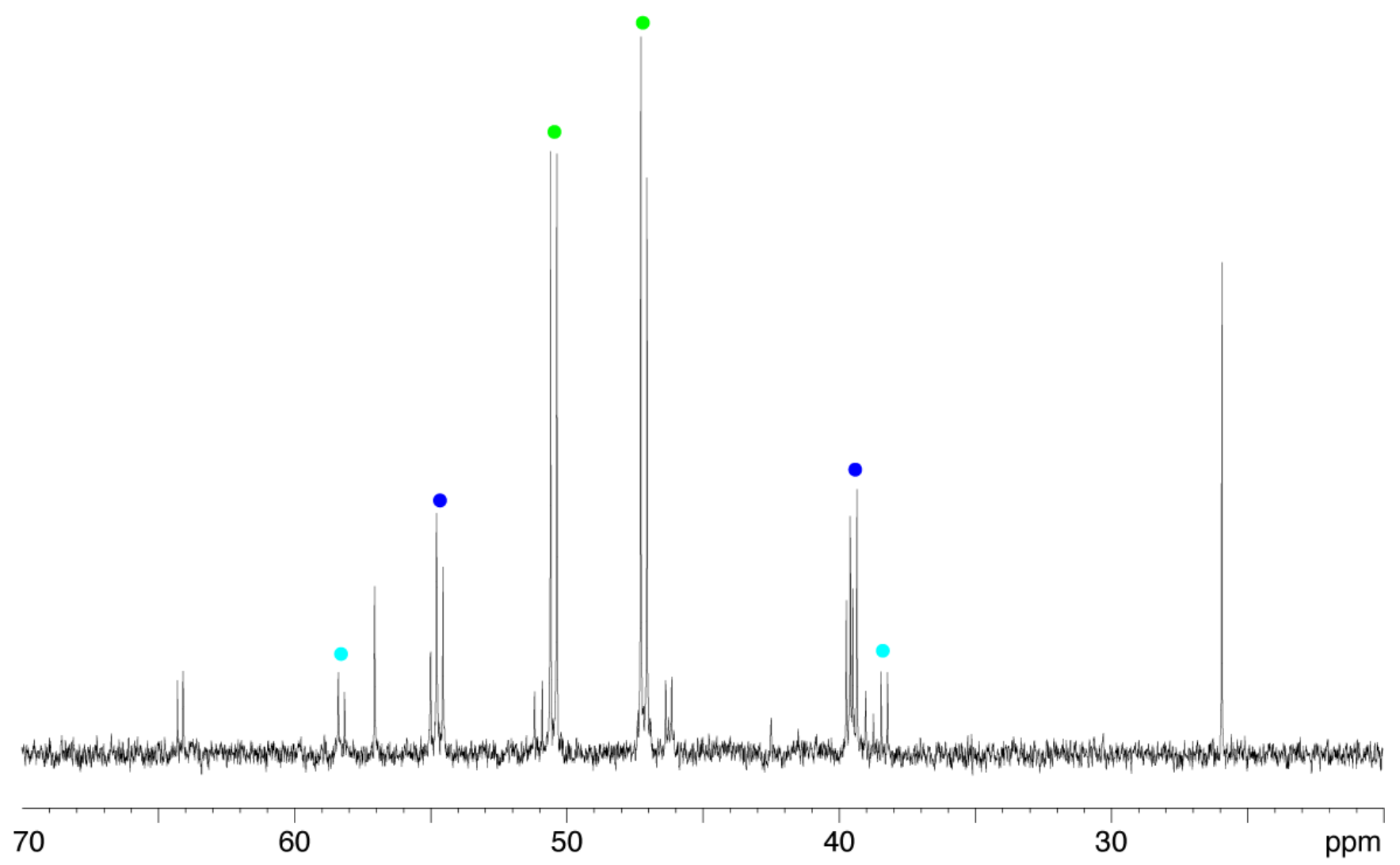

Figure S30. Catalyst resting state resulting from mixture of acrylonitrile, $\mathrm{HPPh}_{2}, 10 \mathrm{~mol} \% \mathrm{7}$, and 10 mol\% DBU after $24 \mathrm{~h}$ (entry 2, Table 2).

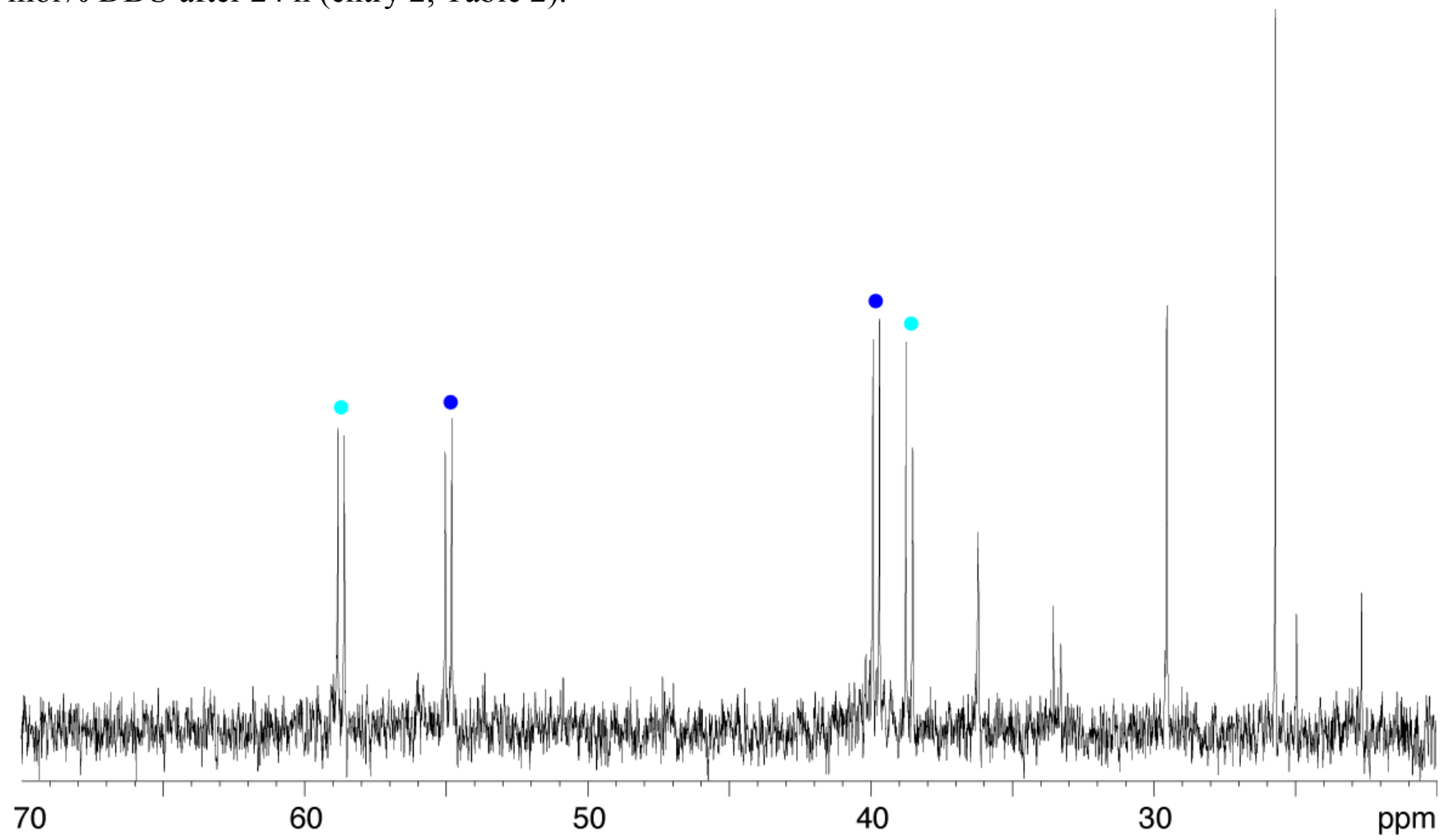

Figure S31. Catalyst resting state resulting from mixture of acrylonitrile, $\mathrm{HPPh}_{2}, 10 \mathrm{~mol} \% \mathbf{8}$, and 10 mol\% DBU after $24 \mathrm{~h}$ (entry 3, Table 2). 


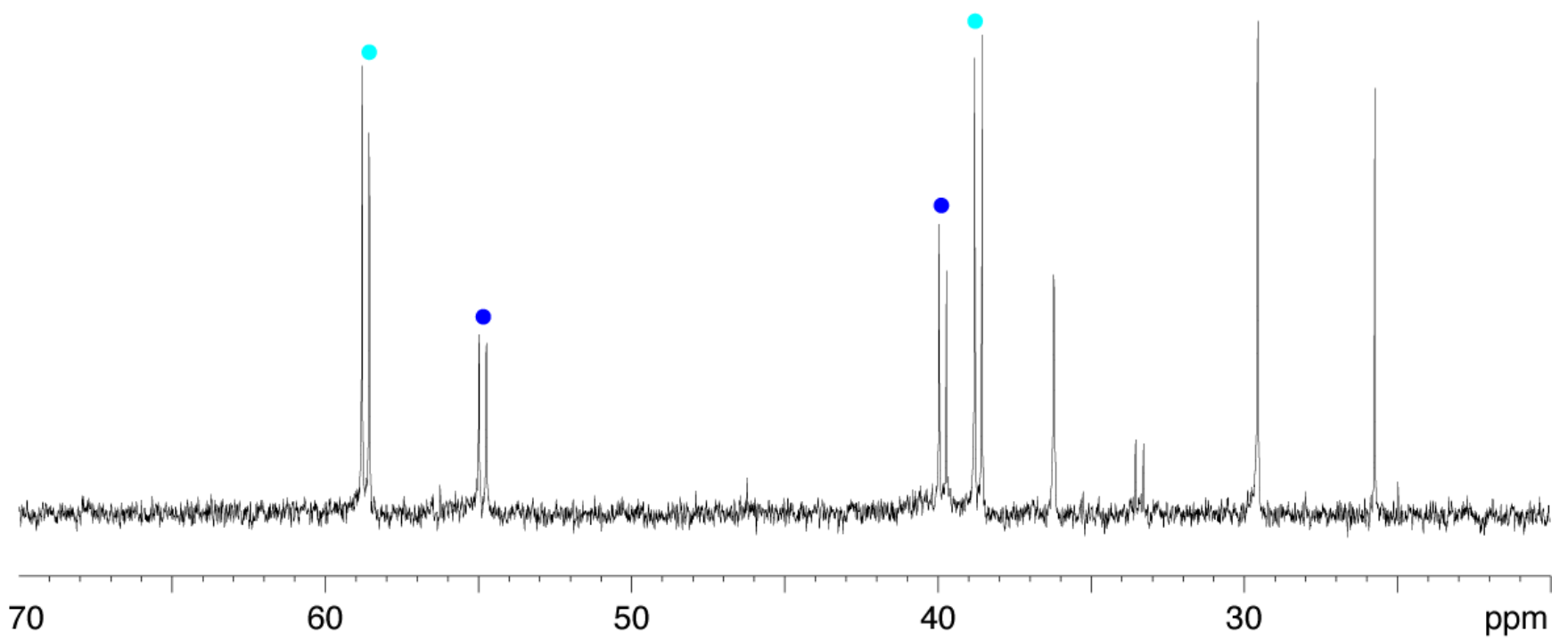

Figure S32. Catalyst resting state resulting from mixture of acrylonitrile, $\mathrm{HPPh}_{2}, 10 \mathrm{~mol} \% \mathbf{9}$, and 10 mol\% DBU after $24 \mathrm{~h}$ (entry 4, Table 2).

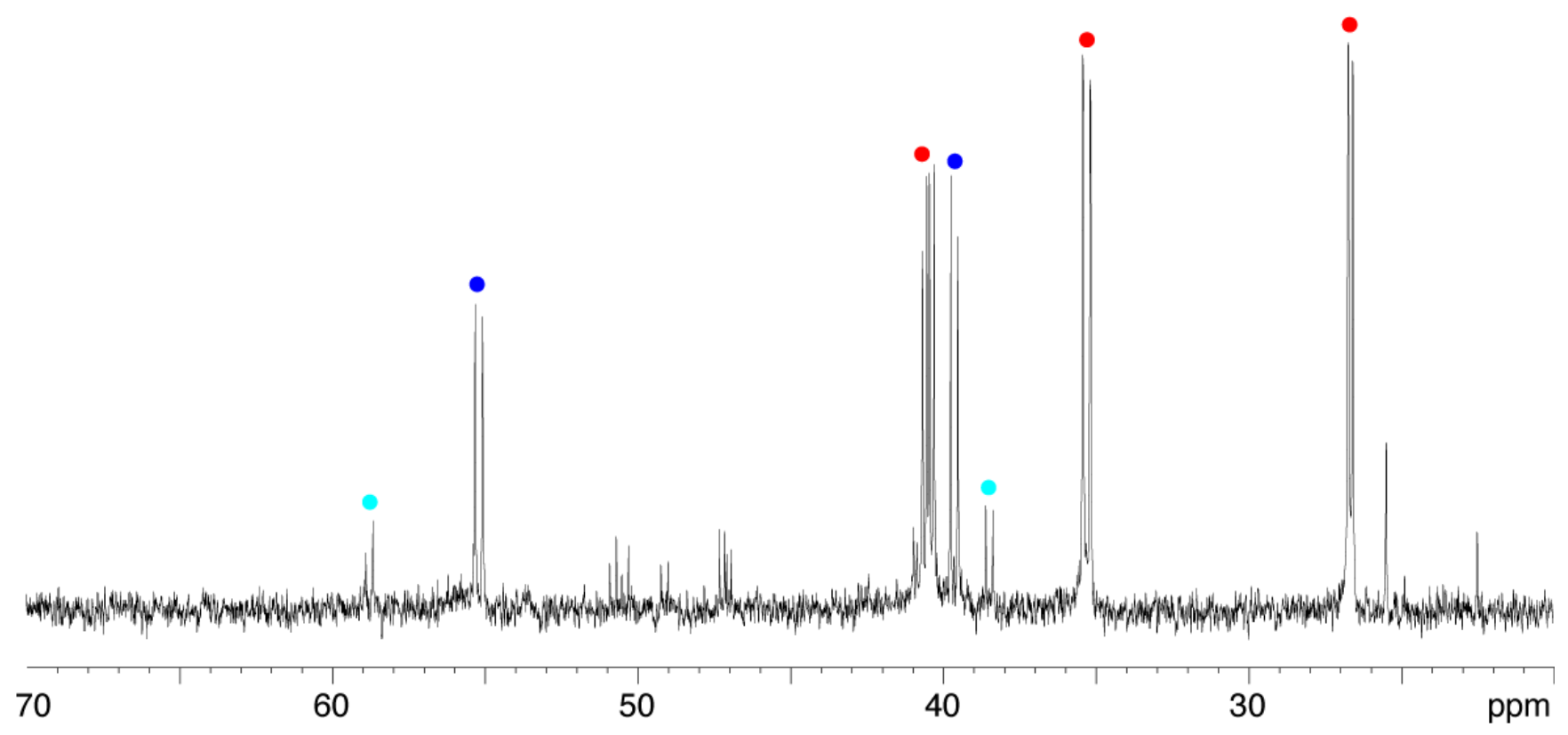

Figure S33. Catalyst resting state resulting from mixture of acrylonitrile, $\mathrm{HPPh}_{2}$, and $10 \mathrm{~mol} \% \mathbf{8}$ after $24 \mathrm{~h}$ (entry 1 , Table 2). 


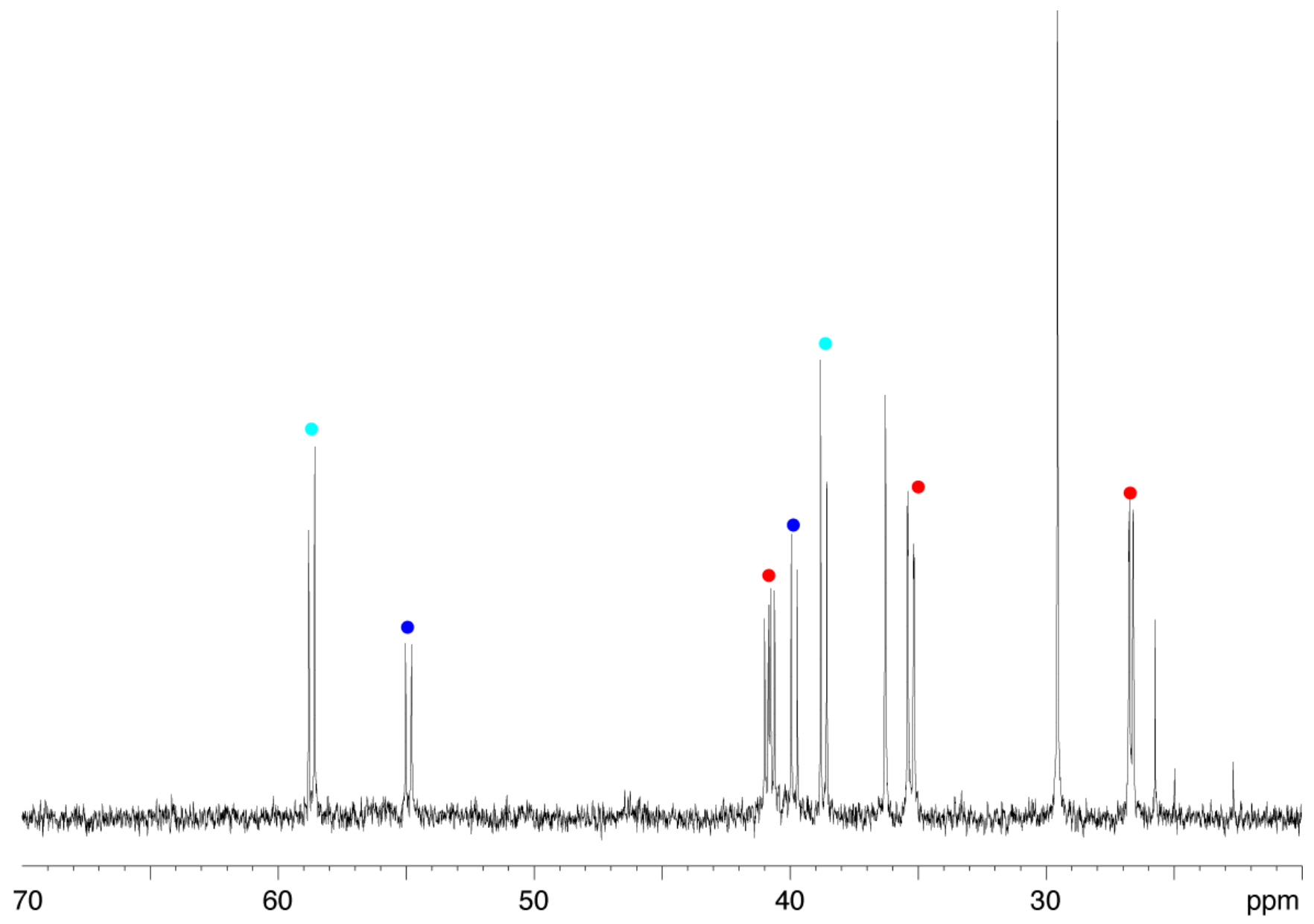

Figure S34. Catalyst resting state resulting from mixture of acrylonitrile, $\mathrm{HPPh}_{2}$, and $10 \mathrm{~mol} \% 9$ after 24 h (entry 1, Table 2). 
${ }^{31} \mathbf{P}\left\{{ }^{1} \mathbf{H}\right\}$ NMR spectra for thermolysis of complex 9:

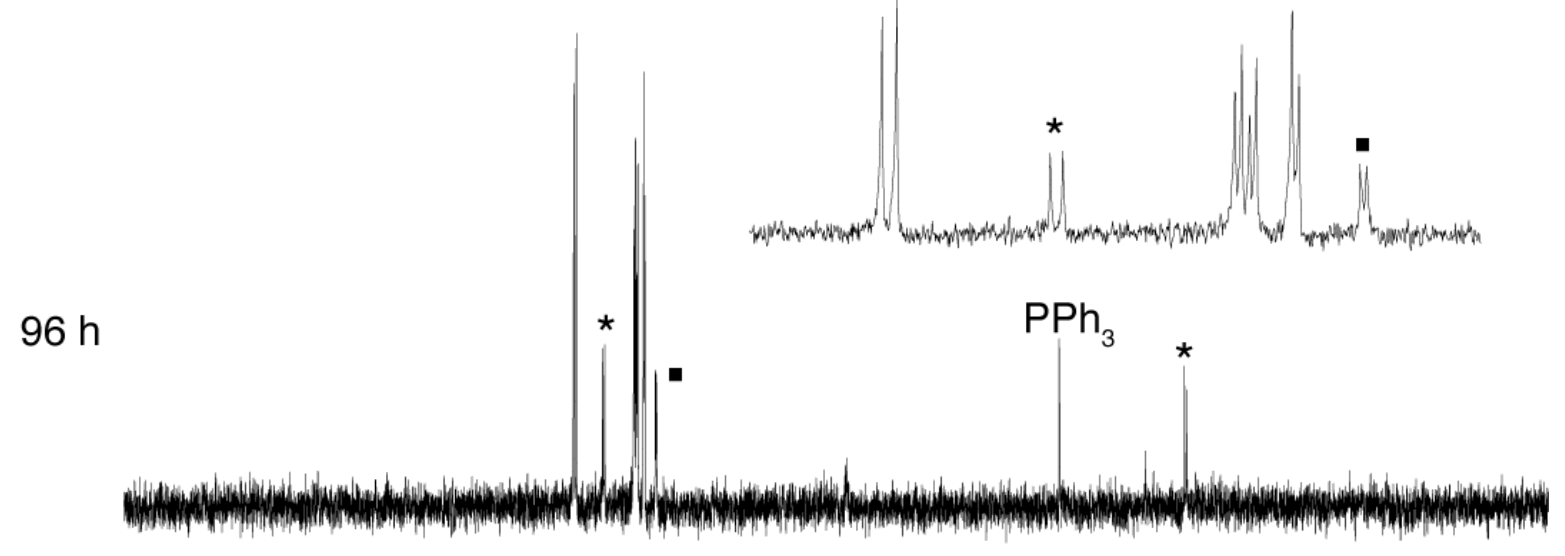

$48 \mathrm{~h}$
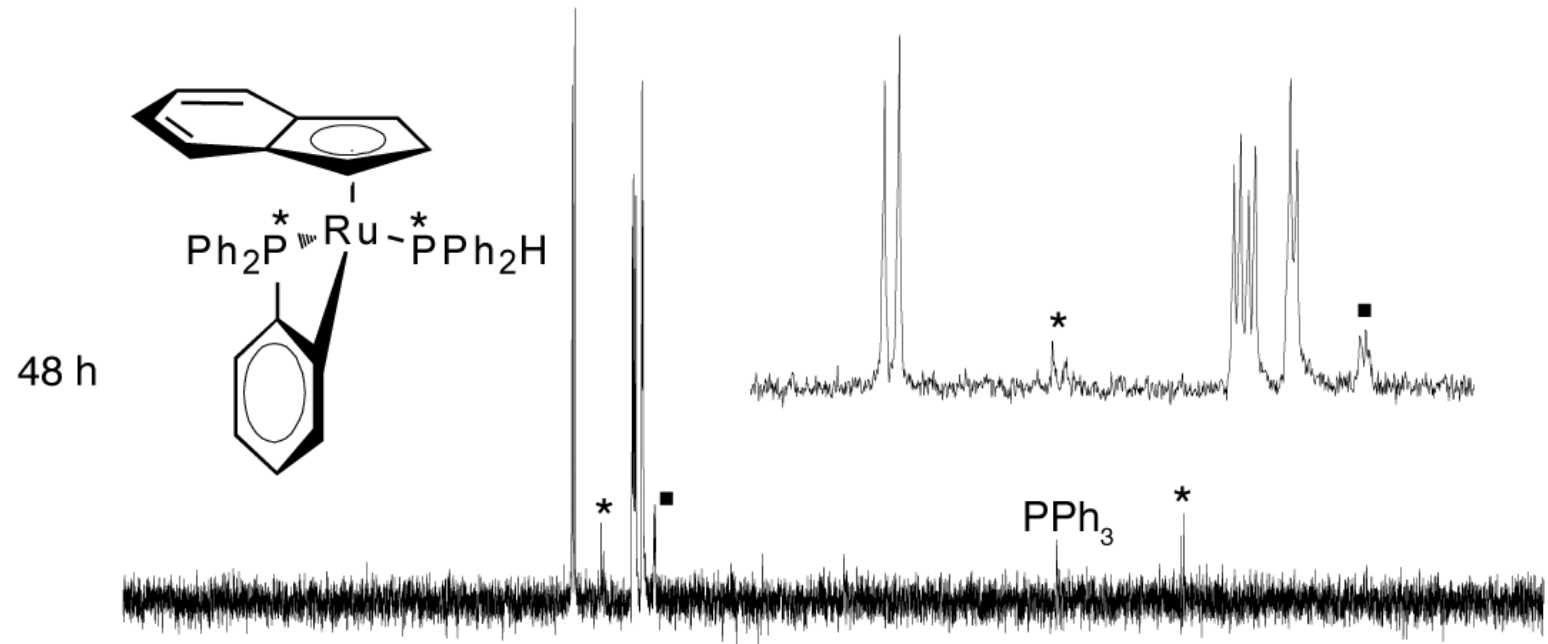

Initial
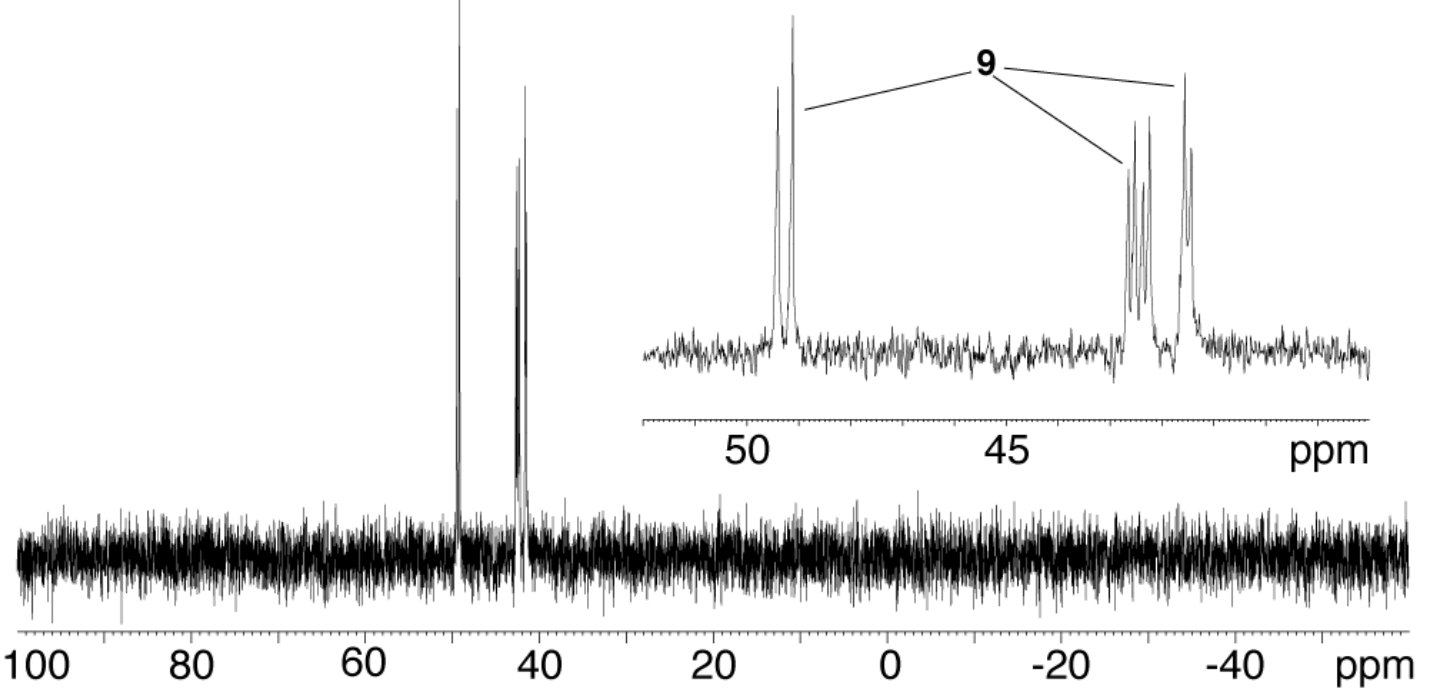

Figure S35. $121.44 \mathrm{MHz}{ }^{31} \mathrm{P}\{1 \mathrm{H}\}$ NMR spectra for a sample of $\left[\mathrm{Ru}\left(\eta^{5}\right.\right.$-indenyl $\left.)\left(\mathrm{PPh}_{2}\right)\left(\mathrm{PPh}_{2} \mathrm{H}\right)\left(\mathrm{PPh}_{3}\right)\right]$ (9) in $d_{8}$-toluene that was heated to $60^{\circ} \mathrm{C}$ for $96 \mathrm{~h}$. (Spectra obtained at RT.) The "*" mark the orthometallation product $\left[\mathrm{Ru}\left(\eta^{5}\right.\right.$-indenyl $\left.)\left\{\mathrm{k}^{2}-\left(o-\mathrm{C}_{6} \mathrm{H}_{4}\right) \mathrm{PPh}_{2}\right\}\left(\mathrm{PPh}_{2} \mathrm{H}\right)\right]$. The black square marks a doublet tentatively assigned to $\left[\mathrm{Ru}\left(\eta^{5}\right.\right.$-indenyl $\left.)\left(\mathrm{PPh}_{2}\right)\left(\mathrm{PPh}_{2} \mathrm{H}\right)_{2}\right]$ (the corresponding triplet is presumably buried beneath signals due to remaining 9 in the sample). 


\section{${ }^{1} \mathrm{H} /{ }^{31} \mathrm{P} \mathrm{HMBC}$ characterization of $\mathrm{CyPCH}_{2} \mathrm{CH}_{2} \mathrm{CN}$ :}

We found no prior literature reports of spectroscopic data for this compound, so we repeated the hydrophosphination of acrylonitrile by $\mathrm{HPCy}_{2}$ catalyzed by complex $\mathbf{6}$ on a slightly larger scale, to separate and analyze more carefully the putative hydrophosphination product.

$\mathrm{HPCy}_{2}(1.0 \mathrm{M}$ in THF, $0.4 \mathrm{~mL}, 0.4 \mathrm{mmol})$, acrylonitrile (1.0 M in THF, $\left.0.4 \mathrm{~mL}, 0.4 \mathrm{mmol}\right)$, and DBU $(0.1 \mathrm{M}$ in THF, $0.4 \mathrm{~mL}, 0.04 \mathrm{mmol})$ were added to $6(60 \mathrm{mg}, 0.04 \mathrm{mmol})$ in a Schlenk flask. The mixture was stirred for $6 \mathrm{~h}$, then the solvent was removed under vacuum. The resulting red oil was eluted through a Florisil column to remove Ru-containing species. Solvent was removed under vacuum from the eluted mixture, and the resulting clear oil was dissolved in $d_{6}$-benzene.

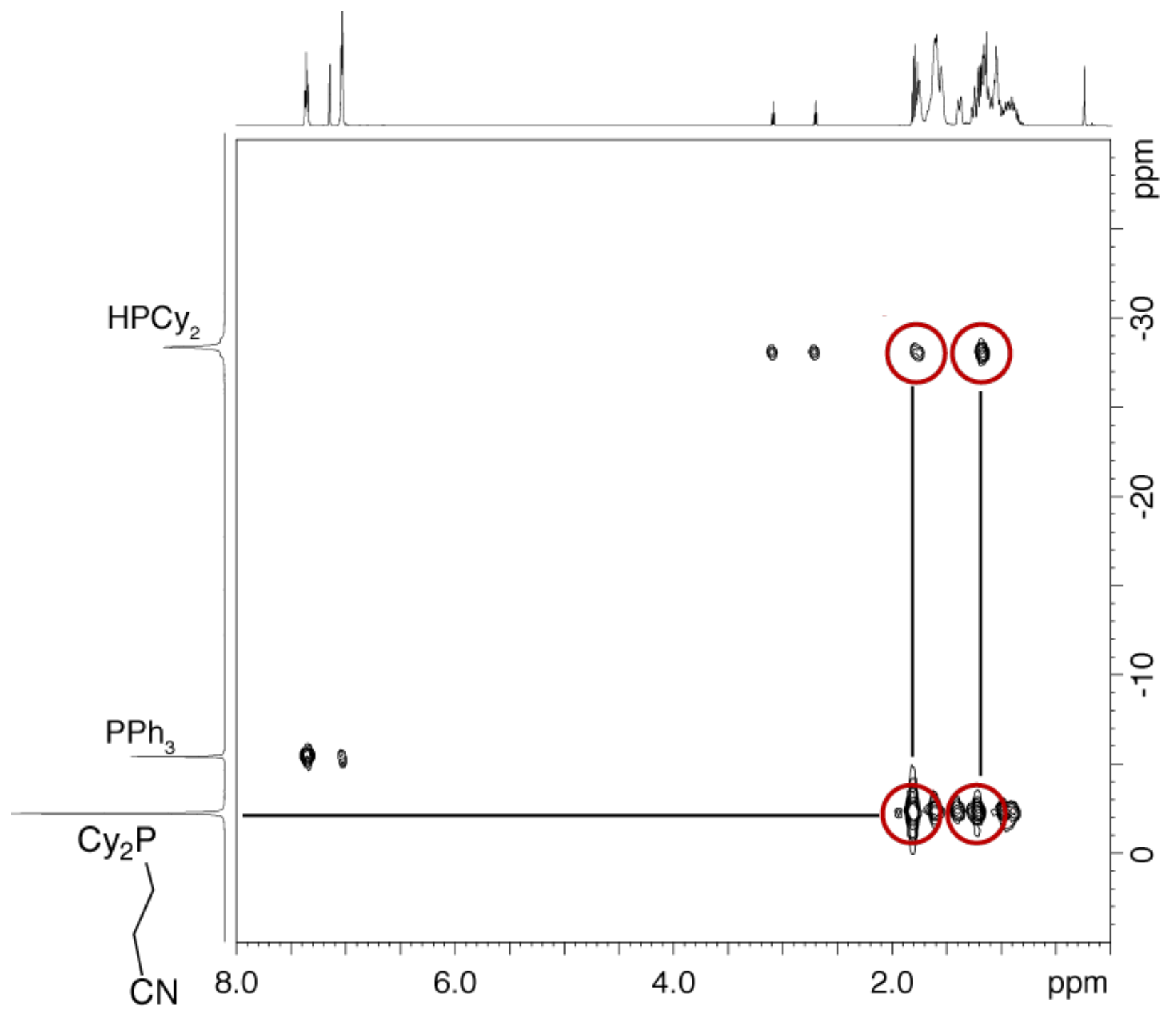

Figure S36. ${ }^{1} \mathrm{H} /{ }^{\beta 1} \mathrm{P}$ HMBC of products isolated from mixture of acrylonitrile, $\mathrm{HPCy}_{2}, 10 \mathrm{~mol} \% \mathbf{6}$, and $10 \mathrm{~mol} \%$ DBU after $6 \mathrm{~h}\left(d_{6}\right.$-benzene, $\left.500.13 \mathrm{MHz}\right)$. Correlations are shown between the putative $\mathrm{Cy}_{2} \mathrm{PCH}_{2} \mathrm{CH}_{2} \mathrm{CN}^{31} \mathrm{P}$ signal and both unique cyclohexhyl $\mathrm{H}_{\text {ipso }}$ signals and other alkyl signals. 